\title{
Peptide Refinement Using A Stochastic Search
}

\author{
Nicole H. Lewis * \\ East Tennessee State University \\ Johnson City, Tennessee \\ Ian L. Dryden \\ University of Nottingham \\ Nottingham, United Kingdom
}

\author{
David B. Hitchcock \\ University of South Carolina \\ Columbia, South Carolina \\ John R. Rose \\ University of South Carolina \\ Columbia, South Carolina
}

\begin{abstract}
Identifying a peptide based on a scan from a mass spectrometer is an important yet highly challenging problem. To identify peptides, we present a Bayesian approach which uses prior information about the average relative abundances of bond cleavages and the prior probability of any particular amino acid sequence. The proposed scoring function is composed of two overall distance measures, which measure how close an observed spectrum is to a theoretical scan for a peptide. Our use of our scoring function, which approximates a likelihood, has connections to the generalization presented by Bissiri et al. (2016) of the Bayesian framework. A Markov chain Monte Carlo algorithm is employed to simulate candidate choices from the posterior distribution of the peptide sequence. The true peptide is estimated as the peptide with the largest posterior density.
\end{abstract}

KEYWORDS: Stochastic Search, Bayesian Methods, Markov Chain Monte Carlo, Peptide Identification, Tandem Mass Spectrometry

\section{Introduction}

Proteomics involves the analysis of proteins, particularly their structure, function, abundances, variations, and modifications. In proteomics, scientists begin with the protein and work backwards to determine the gene that is responsible for its production. Proteins are constantly changing and vary with health or disease while a genome remains relatively static. Issues arise in protein identification when an organism's genome has not been sequenced, more specifically in microbial samples. Only $1 \%-10 \%$ of microbes found in the ecosystem can be cultured. There are countless other microbes that have not been identified and, of the microbes that have been cultured, some will show evidence of post-translational modifications. These post-translational modifications cannot be calculated from the genome (Rose et al., 2010). The area of environmental proteomics has not been fully developed and being able to correctly identify these microbes via protein identification is of great importance especially in ecological samples such as soil and water samples (Schulze, 2004). In clinical proteomics, scientists commonly search for proteins or groups of proteins to help diagnose types of cancers, diseases, or viruses with the goal of early diagnosis. These proteins or groups of proteins can be biomarkers for a disease; see Wulfkuhle et al. (2003), Diamandis (2004), and Visintin et al. (2008). Correctly identifying proteins will also aid in the advance of clinical proteomics.

Current methods for identification of proteins have limitations. With a limited number of known genome sequences, noisy data, and incomplete ion sequences, the accuracy of protein identification requires improvement. In this paper, we describe a Bayesian approach, which aims to improve the identification of proteins.

We employ a Bayesian stochastic search approach to protein identification. We use the prior knowledge of abundances of bond cleavages and the probability of any particular amino acid sequence. Our scoring function combines two

\footnotetext{
${ }^{*}$ Nicole H. Lewis, Department of Mathematics and Statistics, East Tennessee State University, Johnson City, TN 37614 (email: lewiscn2@etsu.edu)
} 
measures of distance that measure the closeness of each observed mass-to-charge ratio $(\mathrm{m} / \mathrm{z})$ value from a sample to an $m / z$ value in a theoretical scan of a peptide. A Markov chain Monte Carlo (MCMC) scheme is utilized to simulate candidate peptides from the posterior distribution, and the peptide with the largest posterior probability is estimated as the true protein. Our approach also allows one to rank the top candidate peptides by their estimated posterior probabilities.

The data come from the Pacific Northwest National Laboratory (PNNL) and can be publicly accessed online for download (Ansong et al., 2011) and is produced by a LTQ Orbitrap yielding doubly charged tryptic peptides. For each peptide, there is a set of $\mathrm{m} / \mathrm{z}$ values with corresponding intensity values.

\subsection{Mass Spectrometry}

There are several methods for obtaining the proteomic profile of a sample. With technological advances, mass spectrometry methods are now more commonly used. Tandem mass spectrometry $(M S / M S)$ is a two-stage mass spectrometry process that allows examination of individual ion fragmentation from a group of ions. Tandem mass spectrometry is used with an assortment of instruments and scan modes.

Peaks can be identified by plotting the intensities versus a horizontal index, which in proteomic analysis is the $m / z$ value. These peaks characterize the peptide in the sample. The final data spectrum is the line plot of pairs of intensities and $m / z$ values (Coombes et al., 2007). Figure 1 pictorially shows the spectrum for a given peptide by plotting the intensity values versus their $m / z$ values. [[Figure 1 goes here]]

\subsection{Protein Identification Methods}

Presently, there are few methods for identifying protein sequences. A popular approach searches through a database of peptides and then matches the closest peptide using the observed spectrum. Some common algorithms for database searches are MASCOT and SEQUEST (Xu and Ma, 2006). Another approach is de novo sequencing, in which the peptide sequence is determined by recreating a spectrum using the observed spectrum. PepNovo and Peaks are frequently used de novo algorithms (Frank and Pevzner, 2005; Frank, 2009; Ma et al., 2003). A more recent approach, de novo sequencing via probabilistic network modeling, uses a mixture of the other two. In this approach, the de novo method recaptures short peptide sequences and then the peptide sequences are used to refine the search in the database approach (Frank and Pevzner, 2005).

A major concern of the database search and hybrid method is that they rely on the use of a database of peptides. These methods cannot correctly identify the protein if it is not in the database. Some limitations of both the database search and de novo peptide sequencing are lack of accuracy and certainty of the chosen peptides, chemical noise, overly complex fragments, and incomplete ion sequences (Lubec and Afjehi-Sadat, 2007). We introduce a Bayesian model that will aim to improve on the PepNovo approach by identifying the correct peptide without depending on the database of peptides, but instead using more generic prior information.

\section{Basic Concepts of Fragmentation}

The basic idea of any protein identification method is to match an observed spectrum to a theoretical spectrum of the proposed peptide. It is extremely difficult to identify intact proteins and so the proteins are broken into short peptides and examined separately. A peptide is a sequence of amino acids, each of which is represented by one of 20 letters. The theoretical spectrum of a peptide is a set of peaks with the location of each peak at the $\mathrm{m} / \mathrm{z}$ value of each ion type. There are spikes at each peak location and zeros everywhere else. The peptide is broken into pairs of ions, most commonly $b$ and $y$ ions. It is the intensities of these ions that are detected in the mass spectrometer. We classify an ion as a $b$ ion if the charge is maintained on the $\mathrm{N}$-terminus, where the $\mathrm{N}$-terminus refers to the beginning of a peptide that is terminated by an amino acid with a free amine group. In order for an ion to be detected, the ion must have a charge of at least one. The $y$ ion is the complement of the $b$ ion. Thus, it is the end of the peptide where the charge is 
maintained on the $\mathrm{C}$-terminus, where the $\mathrm{C}$-terminus refers to the end of a peptide that is terminated by a free carboxyl group $(-\mathrm{COOH})$ (IUBMB, 1992, p. 48).

To find the theoretical spectrum, one must first split the true peptide sequence into all possible ion combinations. In practice, we use only the $b$ and $y$ ions, although there are several other less common ions. After the $b$ and $y$ ions are found, the mass of each ion is determined. The mass for any given ion is found by $\sum_{i=1}^{K} m\left(p_{i}\right)+\delta_{\ell}$ where $K$ is the number of amino acids in the ion sequence, $p_{i}$ is the amino acid in the $i$ th position, $m\left(p_{i}\right)$ is the mass of the amino acid in the $i$ th position, $\ell$ denotes the type of ion such that $\ell \in\{b, y\}$, and $\delta_{\ell}$ is the offset value for that particular ion type. In tandem mass spectrometry, the peptide fragmentation is determined by offsets that correspond to ion types. That is, the offsets match up to the peaks in a given spectrum, and thus denote the different ion types created in the given mass spectrometer (Dančík et al., 1999). Since different types of mass spectrometers yield different spectra, Dančík et al. (1999) developed an offset frequency function that does not depend on instrument type and allows one to define the ion types produced by a given mass spectrometer. The offset value for a $b$ ion is 0.85 Daltons (Da) and 18.85 Da for a $y$ ion.

As an example, consider the peptide $Q V M E L L Q$. There are six $b$ ions and six $y$ ions. The first $b$ ion, $Q$, has a mass of $128.059+0.85=128.909 \mathrm{Da}$, and the first $y$ ion, $Q$, has a mass of $128.059+18.85=146.909 \mathrm{Da}$. Continuing with the splitting of the peptide, one obtains the following additional $b$ ions: $Q V, Q V M, Q V M E, Q V M E L$, and QVMELL with masses 227.977, 359.017, 488.060, 601.144, and 714.228 Da, respectively. Similarly, we obtain the following additional $y$ ions: $L Q, L L Q, E L L Q, M E L L Q$, and VMELLQ with masses 259.993, 373.077, 502.120, 633.160 , and $732.228 \mathrm{Da}$, respectively. Therefore, the theoretical spectrum for the peptide $Q V M E L L Q$ is the set of masses: $128.909,227.977,359.017,488.060,601.144,714.2284,732.228,633.160,502.120,373.077,259.993$, and 146.909 Da. Figure 2 shows the theoretical spectrum for the peptide $Q V M E L L Q$ using only the $b$ and $y$ ions and the positions found above are shown on the $(\mathrm{m} / z)$ axis. [[Figure 2 goes here]]

It is important to find the total mass of the peptide because a mass spectrometer will also measure the total mass of the peptide being analyzed. We can use this weight restriction to eliminate peptides that do not have a total mass within a tolerance of the measured mass. The total mass of the peptide is found by $\sum_{i=1}^{K} m\left(p_{i}\right)+$ mass of $\mathrm{H}_{2} \mathrm{O}$, where the mass of the water molecule is $18.010565 \mathrm{Da}$. For data that are doubly charged, the total mass becomes $\sum_{i=1}^{K} m\left(p_{i}\right)+$ mass of $\mathrm{H}_{2} \mathrm{O}+\mathrm{H}$ because of the second proton that is acquired. The mass of one hydrogen molecule is $1.00794 \mathrm{Da}$. Thus the total mass for the peptide $Q V M E L L Q$ is $860.456 \mathrm{Da}$ assuming the data are doubly charged.

\section{A Bayesian Model}

We propose a Bayesian model with the goal of identifying the true peptide based on the observed spectrum. To identify this true peptide, an MCMC algorithm is used to simulate candidate peptide sequences from an approximate posterior distribution. The motivation will be discussed in Section 3.2.

\subsection{Pre-Processing}

Data that are produced from mass spectrometry methods include a lot of noise. Noise is produced from the oscillation of the instrument (Poisson noise), electrical system (Johnson noise), and matrix ions (chemical noise) (Du et al., 2008). Noise can greatly hinder the identification of peptides in samples. Therefore, the observed spectrum first will be thresholded. Peaks with intensity values below a threshold level will be ignored, and our attention will be focused on the $m / z$ values having intensities above the threshold. A distinct threshold value for each integer $m / z$ value, denoted by $\boldsymbol{T}=\left(T_{1}, T_{2}, \ldots, T_{q \star}\right)$, is computed. Here $q^{\star}$ denotes the total number of $m / z$ values. We define signal peaks as the $m / z$ values corresponding to the $b$ and $y$ ions for a candidate peptide, and we define noise peaks broadly as all other $m / z$ values in the data. The mass spectrometer does not always capture all peaks at the beginning and the end of the spectrum. Thus using only a constant threshold could remove peaks that are truly signal (not noise) peaks. Therefore, we threshold using a weighted average of constant and moving thresholds. The data used in our method consist of the retained $m / z$ values. We will be assuming a Laplace noise structure with details in Section 5.1, which motivates the following approximate model. 


\subsection{Scoring Function}

For our approximate Bayesian model, we first specify a scoring function, which gives a measure of how well the observed spectrum and theoretical spectrum agree. If a candidate peptide's theoretical spectrum does not align well with the observed spectrum, an overall goodness of fit measure will penalize the candidate peptide. Even after thresholding, we still expect there to be noise peaks in the data set and therefore, we incorporate another overall goodness of fit measure that will penalize a candidate peptide when the observed spectrum shows many noise peaks which do not correspond to the $m / z$ values of the candidates theoretical spectrum. We do know that the mass spectrometer does not always capture every signal peak. Hence, we include an indicator function in our scoring function that signifies the presence or absence of a peak. Our scoring function is an approximation to a generative likelihood with details found in Section 5.1. Our method involves treating the scoring function as if it was a likelihood, even though it is actually an approximate likelihood.

We propose a scoring function of the form

$$
L\left(\boldsymbol{X} \mid \boldsymbol{\theta}, \boldsymbol{\eta}, \kappa_{1}, \kappa_{2}\right) \propto \kappa_{1}^{2 p} \exp \left(-\kappa_{1} S_{1}\right) \kappa_{2}^{t-s} \exp \left(-\kappa_{2} S_{2}\right)
$$

where our parameter is vector $\boldsymbol{\theta}=\left(\tau_{1}^{b}, \ldots, \tau_{p}^{b}, \lambda_{1}^{b}, \ldots, \lambda_{p}^{b}, \tau_{1}^{y}, \ldots, \tau_{p}^{y}, \lambda_{1}^{y}, \ldots, \lambda_{p}^{y}\right), \boldsymbol{X}$ contains the observed set of $\mathrm{m} / z$ values for a particular spectrum, and $\boldsymbol{\eta}$ represents the string of amino acids for the candidate peptide. $S_{1}$ and $S_{2}$ are functions of $\boldsymbol{\theta}$ and $\boldsymbol{X}$ that are defined below. The other parameters are defined as

- $s$ is the combined number of $b$ and $y$ ions for the candidate peptide

- $p$ is the number of $b$ ions (or equivalently the number of $y$ ions)

- $t$ is the number of peaks in a given candidate peptide

- $\tau_{i}^{b}$ and $\tau_{i}^{y}$ are the $m / z$ values for the $b$ and $y$ ion of the candidate peptide

- $\lambda_{i}^{b}$ and $\lambda_{i}^{y} \in\{0,1\}$ are indicator functions that signify whether the $i$ th $b$ or $y$ ion has a corresponding observed peak, where $i=1, \ldots, p$

- $\kappa_{1}$ and $\kappa_{2}$ represent weights, which play the role of concentration parameters that control how tightly concentrated the observed peaks are around their corresponding true peaks (see Section 5.1 for more details).

Here, $\lambda_{i}^{b}=1$ denotes the presence and $\lambda_{i}^{b}=0$ denotes the absence of a $b$ ion at position $i$. Similarly, $\lambda_{i}^{y}=1$ denotes the presence and $\lambda_{i}^{y}=0$ denotes the absence of a $y$ ion at position $i$.

Let $x_{j}, j=1, \ldots, n_{T}$ be the observed peaks that are above the threshold $\boldsymbol{T}$ where $n_{T}$ represents the number of peaks that are above the threshold $\boldsymbol{T}$. We will partition these observed peaks into two sets of signal and noise peaks. In particular let $\mathcal{S}$ denote the set of observed peaks above the threshold $\boldsymbol{T}$ which are each closest to one of the peaks $\tau_{i}^{k}$ of the candidate peptide spectrum, $i=1, \ldots, p$ and $k \in\{b, y\}$. We call $\mathcal{S}$ the set of observed signal peaks. Let $\mathcal{N}$ be the remaining observed peaks above the threshold $T$ that are not in $\mathcal{S}$, and we call $\mathcal{N}$ the set of observed noise peaks. The goodness of fit measures of the candidate spectrum relative to the observed spectrum are

$$
\begin{aligned}
S_{1} & =\sum_{i=1}^{p}\left(\lambda_{i}^{b} \min _{j \in \mathcal{S}} d\left(x_{j}, \tau_{i}^{b}\right)+\lambda_{i}^{y} \min _{j \in \mathcal{S}} d\left(x_{j}, \tau_{i}^{y}\right)\right) \\
S_{2} & =\sum_{j \in \mathcal{N}} \min _{i, k}\left|x_{j}-\tau_{i}^{k}\right|
\end{aligned}
$$

and $d\left(x_{j}, \tau_{i}^{k}\right)=\min \left\{\left|x_{j}-\tau_{i}^{k}\right|, \delta\right\}$. We believe that (even allowing for a cushion beyond the usual 0.5 Da threshold, discussed further in Sections 4 and 6, for classifying an observed peak as a true peak) no observed peak will lie beyond $3 \mathrm{Da}$ from the corresponding observed peak. Hence, we choose $\delta=3$. This choice works well in practice; note that choosing $\delta$ much larger than this would inflate $S_{1}$ and worsen the performance.

If $\lambda_{i}^{y}=1$, then $S_{1}$ measures the sum of minimum absolute distances between the closest observed $\mathrm{m} / z$ above a threshold and each $m / z$ peak value of the candidate peak value, while $S_{2}$ measures the sum of minimum absolute distances between each observed $m / z$ value above a threshold and the closest candidate peak $m / z$ values. That is, $S_{1}$ measures the closeness of the nearest observed peak to each candidate $b$ ion or $y$ ion, and $S_{2}$ measures the closeness 
of the nearest candidate peak to each observed peak. Note $S_{1}$ is low when the candidate peaks are close to observed peaks, and $S_{2}$ is low when the noise peaks are close to the candidate peaks or if there are fewer noise peaks. When all peaks for the candidate peptide are very close to observed peaks that are above the threshold, then $\exp \left(-S_{1}\right)$ is high. When all the observed peaks are close to candidate peaks, $\exp \left(-S_{2}\right)$ will be high, so that $\exp \left(-S_{1}\right)$ and $\exp \left(-S_{2}\right)$ represent sensitivity and specificity.

Our method has some interesting connections to other Bayes-like approaches. Although our procedure is different from approximate Bayesian computation (ABC), the fact that we are attempting to get close to the similarity scores in the true unobtainable likelihood has similarities with $\mathrm{ABC}$, although there is no simulation in our procedure but rather just a single evaluation of the approximate similarity scores.

An appealing recent work by Bissiri et al. (2016) presents an innovative idea for generalizing the Bayesian paradigm of updating prior beliefs based on observed data. That article gives a framework in which the role of the data is characterized by a loss function involving the data and the parameters (akin to the loss function considered in decision theory). This loss function could be a negative log-likelihood as in the traditional Bayesian setup, but it also could take other forms, so that the practitioner need not specify a formal likelihood as a model for the data. Using this general loss function along with the prior, the prior beliefs are updated to posterior beliefs after the data are observed.

Our method can be connected to this general updating framework nicely, since our scoring function often will not correspond to a generative data model (except in the special case of Laplace noise, when our scoring function in Equation 1 approximates the generative model given later in Equation 10). The negative logarithm of our scoring function in Equation 1 can, however, be viewed as a loss function that measures the closeness of the observed data to a proposed parameter structure. Based on this closeness, the prior belief about the parameter structure (which characterizes the true nature of the peptide) is updated over the steps of the MCMC process, with our eventual goal being the selection of the "best" parameter structure given the posterior belief, which corresponds to the goal in the Bissiri et al. (2016) framework.

\subsection{Priors}

Huang et al. (2004) estimated the average bond cleavage abundance for each amino acid pair for both the $b$ and $y$ ions for gas-phase dissociation spectra. Collision-induced dissociation (CID) fragments the peptides even further during the gas phase in the mass spectrometry process. A cleavage occurs when the peptide bond fragments during collision induced dissociation, and a cleavage pair is the $b$ and $y$ ion pair that are present in the peptide. For example, take the peptide $Q V M E L L Q$. Recall from Section 2 that $Q V$ is one of the six $b$ ions of the peptide $Q V M E L L Q$ and the complement to that $b$ ion is the $y$ ion $M E L L Q$. These complementary ions are a result of the cleavage between the amino acids $V$ and $M$. This information from Huang et al. (2004) will give us insight about when we expect to see cleavages in the pairs of amino acid residues, and thus we use this information to develop prior information about cleavage pair abundance for our Bayesian approach to identify the true peptide.

\subsubsection{Cleavage Prior}

The cleavage pair abundance prior, denoted $\pi(\boldsymbol{\lambda} \mid \boldsymbol{\beta}, \boldsymbol{\gamma}) \equiv \pi(\boldsymbol{\lambda})$ is defined as:

$$
\pi(\boldsymbol{\lambda})=\prod_{i=1}^{p} P\left(\lambda_{i}^{b}, \lambda_{i}^{y}\right)
$$

with

$$
\begin{aligned}
P\left(\lambda_{i}^{b}=\lambda_{i}^{y}=1\right) & =\rho_{i}^{b y} \times \gamma_{i} \times \beta_{i} \\
P\left(\lambda_{i}^{b}=1, \lambda_{i}^{y}=0\right) & =\rho_{i}^{b y} \times\left(1-\gamma_{i}\right) \times \beta_{i} \\
P\left(\lambda_{i}^{b}=0, \lambda_{i}^{y}=1\right) & =\rho_{i}^{b y} \times \gamma_{i} \times\left(1-\beta_{i}\right) \\
P\left(\lambda_{i}^{b}=\lambda_{i}^{y}=0\right) & =1-\rho_{i}^{b y}+\left[\rho_{i}^{b y} \times\left(1-\gamma_{i}\right) \times\left(1-\beta_{i}\right)\right]
\end{aligned}
$$


where $\boldsymbol{\lambda}=\left(\boldsymbol{\lambda}^{b}, \boldsymbol{\lambda}^{y}\right)=\left(\lambda_{1}^{b}, \ldots, \lambda_{p}^{b}, \lambda_{1}^{y}, \ldots, \lambda_{p}^{y}\right), \rho_{i}^{b y}$ is the geometric mean of the average relative abundance of bond cleavages of $b$ and $y$ ions for a particular amino acid pair for $i=1, \ldots, p$ derived from Huang et al. (2004), $\gamma_{i}$ is the probability of the presence of a $y$ ion, and $\beta_{i}$ is the probability of the presence of a $b$ ion. Here, $p$ represents the number of cleavage pairs. As a matter of notation, note that our parameter vector $\boldsymbol{\theta}\left(=\boldsymbol{\theta}_{\gamma, \beta}\right)$ depends on the values of $\gamma$ and $\beta$, but our notation will suppress this dependency since $\gamma$ and $\beta$ will remain fixed throughout the algorithm. Note that the $\lambda_{i}^{b}$ 's are modeled as having random marginal Bernoulli distributions with probabilities $\rho_{i}^{b y} \beta_{i}$ and the $\lambda_{i}^{y}$ s are modeled as having random marginal Bernoulli distributions with probabilities $\rho_{i}^{b y} \gamma_{i}$, and $\lambda_{i}^{b}, \lambda_{i}^{y}$ are all mutually independent for $i=1, \ldots, p$. The proof of the $\lambda_{i}^{b}$,s and $\lambda_{i}^{y}$ 's having a marginal Bernoulli distribution can found in the supplementary material. Figure 3 shows the geometric mean of the average bond cleavage abundance for all cleavage pairs of the $b$ and $y$ ions using Figure 1 in Huang et al. (2004). Note that probabilities for a particular amino acid cleavage pair that are too close to zero may force the algorithm to exclude reasonable peptides. In order for our prior to be more inclusive, we use a linear transformation of the scale used in Huang et al. (2004), of the form $\rho=0.49 x+0.67$. Our rescaled distribution has probabilities that range from 0.67 to 1.00. [[Figure 3 goes here]]

\subsubsection{Sequence Prior}

We now want to specify a prior distribution for a particular sequence (or string) of amino acids in a peptide. The probability of any particular amino acid sequence is represented by the string prior, $\pi(\boldsymbol{\eta})$, which quantifies the probability of a sequence of amino acids appearing consecutively in a peptide sequence. For each amino acid pair in the candidate peptide under consideration, we count how often the pair occurs in the set of known peptides from the same species. Then we find the empirical probability of each amino acid pair using our large database of peptides. Note that one could use other databases that do not contain the current peptide to calculate the empirical probability. The string prior is defined as proportional to the geometric mean of $\pi\left(\boldsymbol{\eta}_{F}\right)$ and $\pi\left(\boldsymbol{\eta}_{R}\right)$,

$$
\pi(\boldsymbol{\eta}) \propto \sqrt{\pi\left(\boldsymbol{\eta}_{F}\right) \times \pi\left(\boldsymbol{\eta}_{R}\right)},
$$

where $\pi\left(\boldsymbol{\eta}_{F}\right)$ is the joint probability of any particular amino acid sequence calculated from left to right while $\pi\left(\boldsymbol{\eta}_{R}\right)$ is the joint probability of any particular amino acid sequence calculated in the reverse direction. Note that this is the geometric mean of $\pi\left(\boldsymbol{\eta}_{F}\right)$ and $\pi\left(\boldsymbol{\eta}_{R}\right)$. Here $\boldsymbol{\eta}$ is the ordered sequence of the amino acids in the current peptide under consideration where the length of $\boldsymbol{\eta}$ is the number of amino acids in the candidate peptide, and $\pi(\boldsymbol{\eta})$ is a probability for this particular sequence. Denote a generic peptide sequence by $A_{1} A_{2} \cdots A_{m-1}$, where $m-1$ is the number of amino acids in the peptide sequence, $A_{0}$ denotes the beginning of the sequence, and $A_{m}$ denotes the end of the sequence. For example, consider the peptide TGMSNVSK. For this candidate peptide having 8 amino acids, $m=9 . \pi\left(\boldsymbol{\eta}_{F}\right)$ is calculated by

$$
\pi\left(\boldsymbol{\eta}_{F}\right)=P\left(A_{1}=a_{1}\right) \times \prod_{i=1}^{m-1} P\left(A_{i+1}=a_{i+1} \mid A_{i}=a_{i}\right)
$$

with $P\left(A_{1}=a_{1}\right)=p_{1}, P\left[\left(A_{i}, A_{i+1}\right)=\left(a_{i}, a_{i+1}\right)\right]=p_{i, i+1}$, and therefore $P\left(A_{i+1}=a_{i+1} \mid A_{i}=a_{i}\right)=$ $\frac{p_{i, i+1}}{\sum_{j} P\left[\left(A_{i}, A_{i+1}=\left(a_{i}, j\right)\right]\right.}$ for $j \in\{A, C, \ldots, Y, \ldots\}$ where $a_{i}$ represents the amino acid in the $i$ th position in the peptide sequence and $a_{m}=\ldots$ signifies the termination of a sequence. In a similar manner, $\pi\left(\eta_{R}\right)$ is computed by

$$
\pi\left(\boldsymbol{\eta}_{R}\right)=P\left(A_{m-1}=a_{m-1}\right) \times \prod_{i=0}^{m-2} P\left(A_{i}=a_{i} \mid A_{i+1}=a_{i+1}\right)
$$

with $P\left(A_{m-1}=a_{m-1}\right)=p_{m-1}, P\left(A_{i}=a_{i} \mid A_{i+1}=a_{i+1}\right)=\frac{p_{i, i+1}}{\sum_{j} P\left[\left(A_{i}, A_{i+1}=\left(j, a_{i+1}\right)\right]\right.}$ for $j \in\{A, C, \ldots, Y, \ldots\}$ where $a_{0}=\ldots$ signifies the beginning of a peptide sequence. Note that we assign a minimum non-zero probability, 0.000001 , for each pair, so that all peptide sequences have a strictly positive probability. Figure 4 shows the joint empirical probabilities for all pairs of amino acids. An example demonstrating the calculation of this type of prior probability can be found in the supplementary material. [[Figure 4 goes here]] 


\subsubsection{Prior for $\kappa_{1}, \kappa_{2}$}

The concentration parameters, $\kappa_{1}$ and $\kappa_{2}$, are assumed to have independent $\operatorname{Gamma}\left(a_{1}, b_{1}\right)$ and $\operatorname{Gamma}\left(a_{2}, b_{2}\right)$ prior distributions respectively, which are independent of the other parameters.

\subsection{Posterior}

Treating the scoring function as an approximate likelihood, using Bayes' Theorem, the approximate posterior density can be written as

$$
\begin{aligned}
\pi\left(\boldsymbol{\eta}, \boldsymbol{\lambda}, \kappa_{1}, \kappa_{2} \mid \boldsymbol{X}\right) & \propto L\left(\boldsymbol{X} \mid \boldsymbol{\lambda}, \boldsymbol{\tau}, \boldsymbol{\eta}, \kappa_{1}, \kappa_{2}\right) \times \pi(\boldsymbol{\lambda}) \times \pi(\boldsymbol{\eta}, \boldsymbol{\tau}) \times \pi\left(\kappa_{1}, \kappa_{2}\right) \\
& =L\left(\boldsymbol{X} \mid \boldsymbol{\theta}, \boldsymbol{\eta}, \kappa_{1}, \kappa_{2}\right) \times \pi(\boldsymbol{\lambda}) \times \pi(\boldsymbol{\eta}) \times \pi\left(\kappa_{1}, \kappa_{2}\right),
\end{aligned}
$$

where $\boldsymbol{\lambda}, \boldsymbol{\eta}$, and $\kappa_{1}, \kappa_{2}$ are assumed independent. The set of $m / z$ locations given by $\boldsymbol{\tau}=\left(\tau_{1}^{b}, \ldots, \tau_{p}^{b}, \tau_{1}^{y}, \ldots, \tau_{p}^{y}\right)^{T}$ is determined by the sequence $\boldsymbol{\eta}$, and so $P(\boldsymbol{\tau} \mid \boldsymbol{\eta})=1$. Note that this posterior density is only known up to a constant and the actual form of the posterior density is complicated. Therefore, to obtain the posterior probabilities we use MCMC simulation. Our Bayesian method incorporates prior information about the chance of seeing particular cleavage pairs, and also quantifies the prior probability of any particular specific amino acid sequence. We use this posterior density to estimate the true peptide, with candidate peptides having high posteriors being judged more likely to be the true peptide. Our point estimate of the true peptide is the posterior mode, that is, the candidate peptide (among those visited by the search algorithm) with the highest posterior probability, and the posterior distribution variance provides information about the uncertainty of the estimate. Now and henceforth, when we refer to the "posterior," note that this is an approximation to the true posterior, since our scoring function is an approximation to a true generative likelihood (see Section 5.1 for details).

\section{A Markov Chain Monte Carlo Algorithm}

Our posterior is complicated and so we employ Markov chain Monte Carlo (MCMC) methods to sample the parameters (Tierney, 1994; Robert and Casella, 1999; Andrieu et al., 2003; Sorensen and Gianola, 2002).

\subsection{Initialization}

To find a starting peptide for the MCMC algorithm, we only consider candidates with the overall correct mass (within a tolerance). One option is to use an initial iterative sub-algorithm to obtain a starting peptide. Note the actual mass of the true peptide is available to us from the mass spectrometry data, and so we can dramatically reduce the parameter space by searching for peptides with a mass within a specific tolerance $(0.5 \mathrm{Da})$ of the actual mass. To obtain a random starting point, amino acids are randomly added or removed until a peptide is found that has a mass within a tolerance of the mass of the true peptide.

While using the method above will reduce the space of initial peptides, it may still yield a starting peptide far from the truth if the peptide sequence is long, which could result in our method taking a long time to search for the true peptide. Another option for finding a starting peptide is to use the results from PepNovo (Frank and Pevzner, 2005). PepNovo yields a list of the top 2000 best estimated peptides for the true peptide. We can use a peptide from this list as our starting peptide; still ensuring it will have the correct total mass within a tolerance.

\subsection{Posterior Simulation}

Once the starting peptide is generated, the log of the scoring function value of that peptide is calculated. Call the current peptide $\boldsymbol{\eta}_{\text {curr }}$ (initially this will be the starting peptide). The $\boldsymbol{\beta}$ and $\boldsymbol{\gamma}$ vectors are pre-determined at the 
beginning of the algorithm and are constant throughout the algorithm. Before the algorithm begins, a vector $\boldsymbol{\lambda}_{\text {curr }}$ is generated using the $\boldsymbol{\beta}$ and $\boldsymbol{\gamma}$ vectors.

\section{MARKOV CHAIN MONTE CARLO ALGORITHM}

1. A new peptide is created by randomly replacing one, two, or three amino acids of the current peptide with one, two, or three amino acids. This implies the next candidate peptide will be of the same length or length 1 or 2 shorter or 1 or 2 longer than the current peptide, but will still have a total mass within the tolerance of $0.5 \mathrm{Da}$ of the true mass.

2. Generate a vector $\boldsymbol{\lambda}_{\text {new }}$ using the $\boldsymbol{\beta}$ and $\boldsymbol{\gamma}$ vectors.

3. Generate $\kappa_{1}$ and $\kappa_{2}$ from their full conditional distribution: gamma distributions with the shape parameter $\alpha_{1}=a_{1}+s$ and scale parameter $\beta_{1}=S_{1}+b_{1}$ and shape parameter $\alpha_{2}=a_{2}+(t-s)$ and scale parameter $\beta_{2}=S_{2}+b_{2}$, respectively. Note that the values of $S_{1}$ and $S_{2}$ are based on the current peptide.

4. Compute the unnormalized posterior probability for both the new and current peptide, computed based on the new and current $\boldsymbol{\lambda}$ vectors, respectively. Denote these as $\zeta_{1}$ and $\zeta_{2}$, respectively.

5. Generate $U \sim U(0,1)$. If $U<\left(\frac{\zeta_{1}}{\zeta_{2}} \times \frac{q\left(\lambda_{\text {curr }} \mid \lambda_{\text {new }}\right)}{q\left(\lambda_{\text {new }} \mid \lambda_{\text {curr }}\right)} \times \frac{q\left(\boldsymbol{\eta}_{\text {curr }} \mid \boldsymbol{\eta}_{\text {new }}\right)}{q\left(\boldsymbol{\eta}_{\text {new }} \mid \boldsymbol{\eta}_{\text {curr }}\right)}\right)$, then the new peptide becomes the current peptide, and $\boldsymbol{\lambda}_{\text {new }}$ becomes $\boldsymbol{\lambda}_{\text {curr }}$. Otherwise, both the current peptide and $\boldsymbol{\lambda}_{\text {curr }}$ remain unchanged.

6. Go to 1 .

When exploring large state spaces stochastically, it is important that the algorithm be irreducible: that is, it may visit every potential state with positive probability (Tierney, 1994). To ensure irreducibility, every 1000 steps we generate an entirely new peptide that is independent of the current state. Note that any sequence with the correct mass has positive probability of being generated in this step (Tierney, 1994).

Steps 1 - 6 are repeated for a large number of iterations. The peptide with the largest posterior density is selected as the estimate of the true peptide, and we retain all generated peptides along with their approximate posterior probabilities (up to a constant).

Trace plots of the log posterior and parameters are used to monitor convergence of the algorithm to determine whether the chain has converged to its stationary distribution and whether the chain is mixing well. Examples of these plots are are shown in Lewis (2013) in Figures 7.4 - 7.6.

To calculate the first proposal densities we need to calculate $q\left(\boldsymbol{\lambda}_{\text {curr }} \mid \boldsymbol{\lambda}_{\text {new }}\right)$ and $q\left(\boldsymbol{\lambda}_{\text {new }} \mid \boldsymbol{\lambda}_{\text {curr }}\right)$. Note that $q\left(\boldsymbol{\lambda}_{\text {curr }} \mid \boldsymbol{\lambda}_{\text {new }}\right)=$ $q\left(\boldsymbol{\lambda}_{\text {curr }}\right)$ and $q\left(\boldsymbol{\lambda}_{\text {new }} \mid \boldsymbol{\lambda}_{\text {curr }}\right)=q\left(\boldsymbol{\lambda}_{\text {new }}\right)$ since the new $\boldsymbol{\lambda}$ is generated independently of the current $\boldsymbol{\lambda}$ from the prior distribution, as described in Section 3.3.1.

To calculate the second set of proposal densities we need to calculate $q\left(\boldsymbol{\eta}_{\text {curr }} \mid \boldsymbol{\eta}_{\text {new }}\right)$ and $q\left(\boldsymbol{\eta}_{\text {new }} \mid \boldsymbol{\eta}_{\text {curr }}\right)$. Recall from step 1 of the MCMC algorithm, we always replace either one, two, or three amino acids of the current peptide with either one, two, or three amino acids. Hence there is a $1 / 3$ chance of choosing either one, two, or three amino acids to be replaced. If only one amino acid is chosen to be replaced, then there is a $1 / n$ chance that any particular amino acid will be chosen ( $n$ represents the total number of amino acids in the peptide sequence). If a pair of amino acids is chosen to be replaced, then there is a $1 /(n-1)$ chance that a consecutive pair of amino acids will be chosen. If three consecutive amino acids are chosen to be replaced, then there is a $1 /(n-2)$ chance that any particular triplet of consecutive amino acids will be chosen.

Also, note that the current and new peptide must have a total mass that is within a tolerance of the total mass of the true peptide. After the number of amino acids to be replaced is fixed, a list of single, pairs, and/or triplets of amino acids is generated such that each has a mass within a tolerance of the mass of the amino acid(s) that is to be replaced. Therefore, the probability that a particular single, pair, or triplet is chosen is $1 / m$ where $m$ is the number of singles, pairs, and/or triplets in the list of amino acids that satisfy the weight tolerance. If a pair or triplet is selected from the list, then we must consider all permutations of the pair or triplet. For example, if the pair $A K$ is selected from the list, we then randomly select whether $A K$ or $K A$ is chosen. The probability for choosing a particular permutation of a set of amino acids is $1 / v$ where $v$ is the number of permutations of the set of amino acids.

Examples of the calculations of both proposal densities can be found in the supplementary material. 
Since the parameter space is quite large, simulated annealing is performed to help further explore the parameter space. Simulated annealing incorporates a temperature parameter in the algorithm to allow one to better search the parameter space for the true peptide. High temperature values allow more exploration of the parameter space. Lower temperature restricts the exploration of the parameter space (Kirkpatrick et al., 1983). A large temperature parameter is set for the first $95 \%$ of iterations and a small temperature parameter is set for the last $5 \%$ of iterations. The small temperature is set to 1 to ensure we sample from the posterior and we use $L\left(\boldsymbol{X} \mid \boldsymbol{\theta}, \boldsymbol{\eta}, \kappa_{1}, \kappa_{2}\right)^{1 / T}$ in replace of $L\left(\boldsymbol{X} \mid \boldsymbol{\theta}, \boldsymbol{\eta}, \kappa_{1}, \kappa_{2}\right)$ where $T$ is the temperature parameter.

Stochastic search algorithms with finite state spaces typically satisfy certain theoretical properties more readily than those with an infinite number of states (Tierney, 1994). We fix the size of our state space, because for any given spectrum, the peptide cannot be arbitrarily long. A mass spectrometer always accurately measures the total weight of the true peptide and there is a finite number of residues that produces a peptide of that weight.

Since the number of amino acids in the candidate peptides changes across iterations, at first glance it seems as if the dimensionality of our stochastic search is changing. However, we can consider each candidate peptide as one realization from a very large (but finite) sample space. Since the overall mass of the observed peptide is fixed and known, the longest candidate peptide satisfying this overall mass constraint must contain a fixed, finite number of amino acids. There are a finite number of amino acid sequences that combine to yield this overall mass, and our method searches among this large finite set.

\section{Simulation Study}

In this section, we simulate data based on our scoring function in order to get a better understanding of the tuning parameters, with the goal of recovering the theoretical spectrum more often. Since our algorithm uses only the $m / z$ values that have intensities above a threshold, we generate a spectrum with signal and noise peaks that are already assumed to be above a threshold. For a given peptide, we will know the locations of the true peaks. Denote the true set of peak locations as $\boldsymbol{\tau}=\left(\tau_{1}, \ldots, \tau_{s}\right)$, where $s$ represents the total number of true peaks. Each true peak will then generate a signal peak and a random number of noise peaks that are above the threshold. We use two different noise structures, one using the Laplace distribution and the other using a Poisson process.

\subsection{Laplace Noise Structure}

We first employ the Laplace distribution to simulate noise peaks (Damsleth and El-Shaarawi, 1989; Kemp, 2003). Using the Laplace distribution ensures that we have a generative model, allowing us to generate spectra approximately using our model that is defined in Section 3.2.

Mass spectrometers do not always capture peaks that appear at the beginning or end of the spectrum, causing the rate of noise peaks per signal peak to vary over an observed spectrum. Therefore, before we generate a spectrum, we first split the observed spectrum into three sections. Each section will contain a number of signal peaks, determined to be a percentage of the total signal peaks. Then for each signal peak in each section, a random number of noise peaks will be generated. To explain how to find the number of signal peaks for each section, consider a peptide with $s=20$ true peaks. The first section will contain $s_{1}=20 \times 0.1=2$ signal peaks and the third section will contain $s_{3}=20 \times 0.1=2$ signal peaks. Thus the middle section will contain $s_{2}=20-2-2=16$ signal peaks. The proportions of 0.1 for each boundary section reflect the characteristics of the data sets we have studied. For each section of the spectrum, we use a discrete uniform with parameters $a=0$ and $b$ to determine the number of noise peaks per signal peak to be generated. The values of $b$ depend upon the section of the spectrum. Lower values of $b$ will be chosen for the beginning and ending sections and a higher value of $b$ will be chosen for the middle section. Note that increasing $b$ for each section will cause our data to become noisier. For the first section, the value of $b$, denoted as $b_{1}$, will be $b_{1}=3$. For the middle and third section the value of $b$ will be $b_{2}=10$ and $b_{3}=5$, respectively. These values work well and tend to generate a moderate number of noise peaks.

We then simulate from a Laplace distribution to generate the locations of both the signal peak and noise peaks with fixed parameters $\kappa_{1}$ and $\kappa_{2}$. Increasing $\kappa_{2}$ causes the location of the generated noise peaks to be tightly centered on 
the signal peaks. Decreasing the value of $\kappa_{2}$ causes the location of the generated signal peaks to be shifted from their location on the theoretical spectrum and thus be spread out far from the signal peaks.

The steps to generate a spectrum with Laplace noise structure is as follows:

1. Determine the total number of true peaks, $s$, and compute $\tau$ by finding the $b$ and $y$ ions, based on the given peptide.

2. Simulate signal peaks from a density $f\left(x_{j}\right) \propto \kappa_{1} e^{-\kappa_{1}\left|x_{j}-\tau_{j}\right|}$ for $j=1, \ldots, s$, where $\tau_{j}$ are the elements of $\tau=\left(\tau_{1}^{b}, \ldots, \tau_{p}^{b}, \tau_{1}^{y}, \ldots, \tau_{p}^{y}\right)^{T}$. Note $s=2 p$ is the number of peaks and $p$ is the number of b-ions (or $\mathbf{y}$-ions).

3. Use an indicator function $\lambda_{i}$ with probability function $P(\boldsymbol{\lambda})=\prod_{i=1}^{p} P\left(\lambda_{i}^{b}, \lambda_{i}^{y}\right)$, where $p=s / 2$ is the total number of $b$ ions (or, equivalently, the number of $y$ ions) to determine the presence or absence of each signal peak. $P(\boldsymbol{\lambda})$ was given in Section 3.3.1.

4. For each of the three sections in the spectrum, the number of noise peaks for each signal peak in the section is generated using a discrete uniform.

5. Simulate noise peaks from a density $f\left(x_{j}\right) \propto \kappa_{2} e^{-\kappa_{2}\left|x_{j}-\tau_{j}\right|}$, for $j=s+1, \ldots, n_{T}$, where $\tau_{j}$ are peak locations chosen at random from $\tau$.

The likelihood for the generated set of peaks is of the form

$$
L \propto \kappa_{1}^{s} e^{-\kappa_{1} \sum_{j \in \mathcal{S}} \lambda_{j}\left|x_{j}-\tau_{j}\right|} \kappa_{2}^{t-s} e^{-\kappa_{2} \sum_{j \in \mathcal{N}}\left|x_{j}-\tau_{j}\right|} .
$$

Note the similarity of this expression with the previous scoring function in Equation 1 discussed in Section 3.2, although it is not exactly the same. The first component in the scoring function defined in Section 3.2 sums over the minimum absolute distances between the closest observed peak to a candidate peak and the second component sums over the minimum absolute distance between the nearest candidate peak to each observed noise peak. In Equation 10, the first component just sums over the absolute distances between the closest observed peak to a candidate peak and the second component sums over the absolute distance between the nearest candidate peak to each observed peak. We use the scoring function in Equation 1 instead of Equation 10 because we do not know which observed $m / z$ values will match up with the true $m / z$ values. Although it may be possible to estimate, combinatorically it is not sensible. We do not know which observed peak will match up with the theoretical peak but it is practical to assume the closest $m / z$ value.

In the following simulations, we use the previous scoring function in Equation 1, not Equation 10, for inference.

\subsection{Poisson Noise Structure}

To study the robustness of the method to departures from Laplace noise, we may generate noise peaks using a Poisson process. With this process we can generate noise peaks that are independent of the signal peak locations. As with the Laplace noise structure, the spectrum is split into three sections. To determine the number of noise peaks needed for each section, the total number of $m / z$ values, denoted as $q$, that have intensity values above a specific threshold is first found from the observed spectrum of the true peptide. Then $q$ is split into three values, $\left(q_{1}, q_{2}\right.$, and $\left.q_{3}\right)$, where these values will determine the number of noise peaks needed for each section. Reflecting the processing of the spectrum by the mass spectrometer, the first section of the spectrum will have the fewest noise peaks and the middle section will have the most noise peaks. To illustrate how to find the number of peaks needed for each section, consider a peptide whose spectrum contains $100 \mathrm{~m} / z$ values. For the first section of the spectrum, there will be $q_{1}=100 \times .25=25$ noise peaks that are generated. The third section of the spectrum will have $q_{3}=100 \times .25=25$ noise peaks. The middle section will then have $q_{2}=100-25-25=50$ noise peaks. The proportions 0.25 and 0.75 for each boundary section reflect the characteristics of the data sets we have studied. The peptides ranged in mass from 200 to 2000 Da.To obtain the locations for the noise peaks for each section, the cumulative sum of randomly generated values from an exponential distribution, shifted by a specified value $c$, are found by the following algorithm:

1. Initialize $t=0$.

2. Generate $x \sim \operatorname{Exp}(\theta)$ 
3. Set $t=t+x$.

4. Store $t$ in $\boldsymbol{t}$.

5. Repeat $q_{i}$ times.

6. Compute $t+c$

where $\boldsymbol{t}$ is the vector of noise peaks for section $i$ for $i=1,2,3$. In order for the generated noise peaks to have $m / z$ values in the same range as the observed spectrum, we must set an initial value $c$ to be added to the Poisson process. Recall the amino acid $G$ has the smallest mass of 57.0215 Da. Let $\max _{m z}$ be the largest $\mathrm{m} / z$ value for the observed spectrum. For the first section, the value of $c$ (denoted as $\left.c_{1}\right)$ is found by $c_{1}=57+(\max m z-57) \times 0.1$. The value for $c$ for the middle section (denoted as $\left.c_{2}\right)$ is found by $c_{2}=c_{1}+\left(\max _{m z}-57\right) \times 0.2$ and for the last section $c$ (denoted as $\left.c_{3}\right)$ is found by $c_{3}=c_{1}+c_{2}+\left(\max _{m z}-57\right) \times 0.3$. The proportions $0.1,0.2$, and 0.3 were chosen after experimentation. To demonstrate how to find $c$, consider a peptide whose maximum $m / z$ value is 1150 . The values of $c$ for each section would be the following: $c_{1}=57+(1150-57) \times 0.1=166, c_{2}=166+(1150-57) \times 0.2=385$, and $c_{3}=166+385+(1150-57) \times 0.30=879$.

The full algorithm for obtaining the signal and noise peaks using a Poisson process with a parameter $\theta$ is defined as

1. Determine the total number of true peaks, $s$, and compute $\tau$ by finding the $b$ and $y$ ions, based on the given peptide.

2. Simulate signal peaks from a density $f\left(x_{j}\right) \propto \kappa_{1} e^{-\kappa_{1}\left|x_{j}-\tau_{j}\right|}$ for $i=j, \ldots, s$.

3. Use an indicator function $\lambda_{i}$ with probability function $P(\boldsymbol{\lambda})=\prod_{i=1}^{p} P\left(\lambda_{i}^{b}, \lambda_{i}^{y}\right)$, where $p=s / 2$ is the total number of $b$ ions (or, equivalently, the number of $y$ ions) to determine the presence or absence of each signal peak.

4. For each of the three sections in the spectrum, compute the number of noise peaks for each section of the spectrum.

5. Simulate noise peaks from the algorithm described above.

When using a Poisson process to simulate the location of the noise peaks, we need to choose the value of the fixed parameter $\theta$. Increasing $\theta$ causes clusters of tightly spaced noise peaks. Decreasing the value of $\theta$ produces fewer noise peaks in the generated spectrum and thus it does not imitate the observed spectrum as well.

\subsection{Example 1}

Before simulating the spectrum, we must specify the parameters. We set $\kappa_{1}$ and $\kappa_{2}$ to be 50 and 0.10 , respectively. We also set $\theta=1 / 15$. For the indicator function $\lambda$, we set the first elements of $\boldsymbol{\beta}$ and $\gamma$ to be $p_{b 1}=0.05$ and $p_{y_{1}}=0.10$. We set these probabilities to be low because the mass spectrometer rarely captures the first $b$ ion and first $y$ ion. We set all other elements of $\beta$ and $\gamma$ to be 0.8 .

First consider a peptide with a short amino acid sequence. Consider the peptide TGMSNVSK whose observed spectrum contains $\mathrm{m} / z$ values that range from 123 to $749 \mathrm{Da}$. The total number of true peaks is $s=14$ and so $s_{1}=1, s_{2}=12$, and $s_{3}=1$ using boundary section proportions 0.1 . The total number of $\mathrm{m} / z$ values in the observed spectrum with intensity values above the threshold is $q=75$ and so $q_{1}=19, q_{2}=37$, and $q_{3}=19$.

For each example shown, a table of the 5 best estimated peptides is given along with their corresponding log posterior value. The breakdown of the log posterior is also given to show why the true peptide does not have the highest posterior probability (when that is the case).

We simulate a spectrum with minimal noise and one with substantial noise. For the Laplace case, to decrease the number of noise peaks, we set the values of $b$ for each section of the spectrum to be $b_{1}=b_{2}=b_{3}=2$. To increase the number of noise peaks, we set the values of $b$ for each section to $b_{1}=b_{2}=b_{3}=10$. For the Poisson case, to decrease the number of noise peaks, we decrease the values of $q_{1}, q_{2}$, and $q_{3}$ by 15 and to increase the number of noise peaks in the generated spectrum, we increase the values of $q_{1}, q_{2}$, and $q_{3}$ by 15 . 
After the spectrum is generated, our method described in Section 3.2 is then applied to the simulated spectrum. The starting peptide is GTMSGRSQ, which was obtained from the results from PepNovo when applied to the real data. The algorithm was run for 10000 iterations.

Table 1 shows the posterior mode for the TGMSNVSK example using the simulated spectrum for each of the three noise levels using a Laplace noise structure. Table 2 shows the posterior mode for the TGMSNVSK example using the simulated spectrum for each of the three noise levels using a Poisson noise structure. Both tables provide the estimated log posterior densities with the breakdown of the log posterior. The true peptide is highlighted in bold. Both tables show that when using minimal noise, the true peptide is the top estimated peptide, which ensures our method is performing well in both the Laplace and Poisson case. In cases when the spectrum has more noise, our method was still able to identify the true peptide as among the best choices using either noise structure. With moderate noise, the true peptide is estimated as the best peptide under the Laplace noise structure. Under the Poisson noise structure with moderate noise, the true peptide is estimated as the second best peptide, but notice that the log posterior for the best estimate and the $\log$ posterior for the true peptide are quite similar. [[Table 1 goes here]] [[Table 2 goes here]]

\subsection{Example 2}

Here we set the parameters to be the same as in Section 5.3. We now generate a spectrum for a peptide with a longer amino acid sequence. The generated spectrum is based on the peptide YHFEQSTVTSQPAR whose observed spectrum contains $m / z$ values that range from 235 to $1634 \mathrm{Da}$. The total number of true peaks is $s=26$ and so $s_{1}=3, s_{2}=20$, and $s_{3}=3$ using boundary section proportions 0.1 . The total number of true peaks is $s=26$ and so $s_{1}=3, s_{2}=20$, and $s_{3}=3$. The total number of $m / z$ values in the observed spectrum with intensity values above a threshold is $q=157$ and so $q_{1}=39, q_{2}=79$, and $q_{3}=39$ (up to a constant).

As in Example 5.3, we also simulate spectra with minimal and substantial noise. After the spectrum is simulated, we applied our method to the simulated spectrum. The starting peptide is HYFETDQATSKPVK, which was obtained from the results from PepNovo when applied to the real data. The algorithm was run for 10000 iterations.

Table 3 shows the posterior mode for the YHFEQSTVTSQPAR example using the simulated spectrum for each of the three noise levels under a Laplace noise structure, and Table 4 shows the posterior mode for the

YHFEQSTVTSQPAR example using the simulated spectrum for each of the three noise levels using Poisson noise. Both tables provide the corresponding estimated log posterior densities with the breakdown of the log posterior. The true peptide is highlighted in bold. Table 3 shows when minimal noise is applied, the true peptide again is the top estimated peptide, which shows our method is performing well. When moderate noise is applied, the true peptide is identified as the best estimated peptide. When substantial noise is applied, we see that the true peptide was not identified in the top estimated peptides, because of the additional noise added into the spectrum. Table 4 shows when minimal noise is applied, the true peptide again is the best estimated peptide, confirming that our method is performing adequately well. With moderate noise, the true peptide is estimated as the second best peptide. With substantial noise, the true peptide is among the top estimated peptides. Although the spectrum has more noise, our method was still able to identify the true peptide as being among the best choices in this example. [[Table 3 goes here]] [[Table 4 goes here]]

\subsection{Comparison of Noise Structures}

With moderate noise, our method performed equally well under both noise structures for peptides with both short and long amino acid sequences. With minimal noise, once again our method performed equally well under both noise structures for peptides with short and long amino acid structures. With minimal noise, the true peptide was identified in all cases.

For peptides with short and long amino acid sequences, using either noise structure, our method performed well for all levels of noise tried. The noise generated in the spectrum is not completely realistic since certain peak features like isotopic peaks, adducts, and post-translational modifications are not included in the noise and so the noise generated is somewhat artificial. Although the noise generated in the spectrum may not be realistic, the methods are promising. An advantage of assuming a Laplace noise structure is that our model would be an approximation to a generative model. 
Unlike a discriminative model, a generative model allows one to generate samples from the joint distribution. Generative models are more flexible since they are full probabilistic models of all variables and can be used to simulate values of any variable in the model (Singla and Domingos, 2005). Note our function in Equation 1 used is an approximation to the Laplace model.

\section{Real Data Application}

Most peptides in the PNNL dataset described in Section 1 are of length 8 to 20 amino acids. Our data include some relatively longer peptides due to the type of equipment used to process the data. Recall the equipment used was a LTQ Orbitrap mass spectrometer, which is a hybrid machine composed of a linear ion trap mass spectrometer and the Orbitrap mass analyzer that uses a fast Fourier transform algorithm (Yates et al., 2009). The dataset contains 1,206 peptides with lengths ranging from 7 to 31 amino acids and an average length of 15.16. The data are doubly charged and the total mass for each peptide is given. The dataset contains a set of masses and corresponding intensities with an average intensity value of 50.7 .

We first process the data. We choose to remove the doubly charged parent ion from the dataset. A parent ion is the fragment ion generated in mass spectrometry before the ion is broken apart into further ions. The $m / z$ value of the doubly-charged ion is $\frac{\sum_{i=1}^{K} m\left(p_{i}\right)+1}{2}$. Therefore, we remove the peak at that $m / z$ value. Our proposed method works for pre-processed data. After extensive numerical experimentation, we found that using the 75th percentile to calculate the constant and moving threshold works well. This means we use the observed $m / z$ values in the data that have corresponding observed intensity values above the threshold value in $\boldsymbol{T}$. We looked at several other threshold values to see which optimized the results. As the threshold is decreased, the estimated peptides become less similar to the true peptide. This happens because as we lower the threshold, more noise enters the observed spectrum and the value of $S_{2}$ in the likelihood is greatly increased. Therefore, our algorithm cannot find the true peptide. As the threshold is increased, at a certain point the estimated peptides become less similar to the true peptide. Although there is less noise in the observed spectrum when the threshold is increased, signal peaks may be removed from the observed spectrum with a large threshold. Thus, our algorithm will not be able to correctly to identify the true peptide. Using a threshold of 75\% removes many noisy peaks while still retaining the signal peaks. Tables 7.17 - 7.20 in Lewis (2013) illustrate these results.

The mass spectrometer is not always accurate and this can cause the ion fragments that are detected to be slightly shifted from their theoretical position. Therefore, we use a tolerance level of $0.5 \mathrm{Da}$. That is, we allow the ion peak locations to be up to $\pm 0.5 \mathrm{Da}$ from their theoretical positions. We set the initial components of $\boldsymbol{\beta}$ and $\boldsymbol{\gamma}$ to be $p_{b 1}=0.05$ and $p_{y_{1}}=0.10$. We set these probabilities low because the mass spectrometer rarely captures the first $b$ and $y$ ion. Table 1 in Dančík et al. (1999) provides prior probabilites for observing a $b$ or $y$ ion based on experimental spectra. We used those prior probabilities as initial starting values for $p_{b i}$ and $p_{y_{i}}$. After varying the values of $p_{b i}$ and $p_{y_{i}}$ based on extensive experimentation, setting $p_{b i}$ and $p_{y_{i}}$ to equal 0.80 worked well. Therefore, we set all other $p_{b i}$ and $p_{y_{i}}$ to equal 0.80 for $i=2, \ldots, p$.

We must also specify the hyperparameters, the most critical, in the Gamma prior distribution for $\kappa_{1}$ and $\kappa_{2}$. After extensive numerical experimentation, the values of $a_{1}, b_{1}, a_{2}$, and $b_{2}$ were set to be 5.5, 0.1,3, and 100, respectively. From further experimentation, the large temperature parameter was set to 500 and the small temperature parameter is 1. We believe these values work well for the peptides of moderate size peptides studied in this paper. For extremely large peptides, the values of the hyperparameters may need to be adjusted to higher values. Based on experimentation, a general criterion for large peptides (i.e., comprising roughly 30 or more amino acids) is to use values for the hyperparameters that are 5 times larger than those used for moderately sized peptides.

Different tolerances affect the performance of the method. Using a small tolerance like $0.1 \mathrm{Da}$ hardly allows for any error in the mass spectrometer, so that the observed spectrum would need to be aligned almost perfectly with the theoretical spectrum. A large tolerance like 1.0 Da would allow more room for error but it would expand the parameter space that needs to be searched, which could prevent the algorithm from finding the true peptide in an efficient manner.

Starting from a completely random place is idealistic, but it may not be best in practice since the state space is so large, which would cause the algorithm to run for a much longer number of iterations. Running the algorithm for a large 
number of iterations increases the running time of estimating the peptide. Thus, the results from PepNovo should be used to obtain a starting peptide. We then refine the estimated peptide using our proposed method.

\subsection{Example 3}

Figure 5 is a plot of the observed spectrum for the peptide TGMSNVSK. The theoretical spectrum aligns nicely with the observed spectrum, although there is quite a bit of noise in the center of the graph even after thresholding. [[Figure 5 goes here]]

Using the results from PepNovo, we obtain a starting peptide, TGFAGGVSGA, which has a total mass that is within $0.5 \mathrm{Da}$ of the weight of the true peptide. After 100,000 iterations, our best estimate for the true peptide is $T G M S N V S K$ with a $\log$ posterior density of -15.16 (up to a constant). Table 5 shows the top estimated peptides for the TGMSNVSK example, with their corresponding estimated log posterior densities and the breakdown of the $\log$ posterior (log of the scoring function valueand $\log$ priors). The true peptide is estimated as the best having the largest log posterior density. [[Table 5 goes here]]

To ensure our method is obtaining similar results for various starting peptides, consider results from using different starting peptides that we obtain from PepNovo: SAMYHSK, TGAFGRSK, and GTFANEGK. Table 6 shows the top estimated peptides along with their corresponding log posterior densities for the above starting peptide values. The results are similar and that the true peptide (highlighted in bold in the table) is captured as the best estimated peptide in all three cases. The log posterior densities are also similar in the three cases. [[Table 6 goes here]]

\subsection{Example 4}

Figure 6 is a plot of the observed spectrum for the peptide $D L V E S A P A A L K$. The theoretical spectrum aligns nicely with the observed spectrum. [[Figure 6 goes here]]

Using an initial peptide of DLVESY FLK from the PepNovo results and 100,000 iterations, we obtain our estimate $D L V E S A P A A L K$ with a log posterior density of 42.90 (up to a constant). We see that the true peptide is estimated as the best. Table 7 shows the top estimated peptides for the DLVESAPAALK example along with the breakdown of the $\log$ posterior. [[Table 7 goes here]]

Consider the results from using different starting peptides that we obtain from PepNovo: DLVTDAPAAIQ, $L D V T D A P A A L K$, and $L D V E T G P A A I Q$. Table 8 shows the top estimated peptides along with their corresponding log posterior densities for the above starting peptide values. Again the true peptide (highlighted in bold in the table) is the best or is among the best choices in each case, and the log posterior densities are also similar in the three cases. [[Table 8 goes here]]

\subsection{Result Comparisons}

To quantify objectively how our method improves relative to its current estimation, we compare our results with those using the PepNovo rank score and the PepNovo score. The comparison is only made in the accuracy of the predicted peptide and not in the speed of the computation. Since our method can identify peptides that are not found in a peptide database, we make no comparisons with SEQUEST and MACSOT. We will not make a distinction between the amino acids $I$ and $L$ because they have identical masses of 113.084. Although PepNovo does not make a distinction between the amino acids $K$ and $Q$ because the difference in their masses is only a minute difference of $0.04 \mathrm{Da}$, we will make the distinction.

Our comparison method uses the minimum number of switches in the amino acid sequence of the peptide needed to obtain the true peptide. Switches are only considered if the total mass remains within $0.5 \mathrm{Da}$ of the total mass of the true peptide. If the best estimated peptide is the truth, then minimum number of switches would be zero. To illustrate this comparison method, consider the true peptide $V S E G Q T V R$ with the estimate $W E G Q T V R$. One can see the only difference from the true peptide is that the estimate begins with $W$ while the true peptide begins with $V S$. Note that the mass of $W(186.079 \mathrm{Da})$ is within $0.5 \mathrm{Da}$ of the mass of $V S(186.1)$ and so the minimum number 
of permissible switches is 1 . If more than 3 switches are needed to obtain the true peptide, we denote the minimum number of switches as $4+$. In both the PepNovo rank score and PepNovo score, the best estimated peptide might not have the same mass as the true peptide. PepNovo does provide the $N-$ Gap, which is the mass gap from the $\mathrm{N}$-terminal to the start of the de novo sequence and the $C-$ Gap, which is the mass gap from the C-terminal to the end of the de novo sequence. While it does provide those mass values, it does not detect the amino acid residues that should correspond to the mass gaps. For example, consider the true peptide DLVESAPAALK with a total mass of $1113.616 \mathrm{Da}$. Using the PepNovo rank score, the best estimated peptide is DNVESLEV, which has a mass of 885.4088 Da. Note that this mass is the sum of masses of each amino acid residue in the sequence and does not include the mass of a water molecule and hydrogen molecule. That is accounted for in the mass gap. The $C-G a p$ value given is $229.029 \mathrm{Da}$ implying there are amino acid residues missing from the end of the de novo sequence whose mass should total 229.029 Da. We cannot look at the minimum number of switches; however, we do know that a peptide with a total mass less than the total mass (outside of the tolerance) of the true protein cannot be the true peptide.

Table 9 displays the best estimated peptides for the PepNovo rank score, the PepNovo score, and our method along with the corresponding true peptide. The minimum number of switches is in parentheses. One can see that in most cases when using the PepNovo rank score, the best estimated peptide does not have the correct total mass. Thus, refining the results of PepNovo, our method allows our estimated peptides to have the correct total mass (within a tolerance). Comparing the results from the PepNovo score and our method of using the Bayes log posterior, the PepNovo score tends to do slightly better for peptides with shorter amino acid sequences. However, for peptides with longer amino acid sequences, our method tends to do better. PepNovo produces very quick (seconds) results but the MCMC algorithm is slower (several minutes). However, we do not see our method as a competitor but more as a refinement. Note that one method does not necessarily work best in every case, yet a combination of two good methods can produce an even better method. Therefore, an avenue to explore in the future is developing a rank score method that will combine our method with PepNovo's method. It would be interesting to compare the number of switches for peptides in order to gauge both the false positive and false negative error rates, although this is an enormous computation. [[Table 9 goes here]]

\section{Discussion}

Proteomics produces large amounts of spectra from mass spectrometry. Issues such as post-translational modifications (PTMs), mutations, and contaminants can cause the spectra to fail to match peptides from a database. Also, there are copious microorganisms such as bacteria and protists that have not been identified and therefore, using a database search to identify these peptides is of very limited use in the case of non-homologous proteins. Of those microorganisms that have been identified, some show evidence of PTMs, which can create complications in the de novo sequencing when comparisons are made between the theoretical spectrum and the observed spectrum. Thus the need for a method of identifying peptides that does not rely on a known database and is not is affected by PTMs is evident.

Another reason for the need for accurate peptide identification is protein sequencing, the method of identifying the true amino acid sequence of a protein. Identifying an entire protein is almost impossible, and so the protein is split into short peptides. Ergo, being able to correctly identify the amino acid sequence of a peptide will aid in identifying the true protein sequencing.

There are limitations in the current methods for protein identification. Our method aims to alleviate such drawbacks of de novo sequencing and database searches. By using a Bayesian approach, we allow prior knowledge of the peptides to help us to find the best estimate of the true peptide. Due to the complexity of our posterior density, we use MCMC simulation to obtain the posterior probabilities. This allows one to approximate the target distribution, in our case the posterior distribution of the unknown peptide sequence. One advantage of our method is that it is not dependent upon known peptides. We hope that our method will obtain more accurate estimates of the true peptide, helping researchers in the field of proteomic research and potentially aiding in identifying microbes. With the study of proteins becoming more important in identifying early stages of diseases (most commonly cancer), it is of great importance to be able to correctly identify these proteins. Conceivably, use of these peptides as biomarkers for diseases could aid in the diagnosis of types of cancers or find better patient treatments. 
Cleveland and Rose (2012) developed a method to identify better peaks using a neural network, which can be used to construct a predictive model that does not require an extensive understanding of peptide fragmentation. Like our method, Cleveland and Rose (2012) concentrate on identifying signal peaks corresponding to $b$ and $y$ ions; they also employ a leveraged neural network (LNN), which is composed of two neural networks used in order to classify peaks. In the first neural network, peak features, such as isotopologues and neutral losses, are found from the data in the spectrum. Then in the second neural network, the results from the first neural network are leveraged as extra features in the second neural network. This process selects peaks with higher precision and reduces the number of peaks in the spectrum, which could make identifying the true peptide more efficient. Recall from Section 2 that other ions can produce peaks in the observed spectrum. Therefore, employing this method could lead to better identification of peaks, and thus will lead to a more accurate theoretical spectrum and ultimately better identification of peptides. For additional information about the LNN, see Cleveland and Rose (2012).

An important aspect for the future development is to refine the Bayesian model. The model can be refined by including more signal peaks: $b-\mathrm{H}_{2} \mathrm{O}, b-\mathrm{NH}_{3}, y-\mathrm{H}_{2} \mathrm{O}, y-\mathrm{NH}_{3}, y^{2}$, isotopic peaks, etc, which could aid in identifying which are signal peaks and which are noise peaks. Adapting the prior to assign probabilities to the length of the peptide is another avenue one could explore. The current prior favors shorter peptide sequences. Peptides with shorter amino acid sequences have higher prior probabilities than peptides with longer amino acid sequences.

There are a few other possibilities for future work: Exploration of the sequence prior distribution could be conducted, although a full exploration would be a massive undertaking since the number of sequences is so vast. The calculated priors could be compared with the observed frequencies to gain an idea of how closely the prior distribution reflects the empirical distribution. A sensitivity analysis in the simulations could be explored. Possible approaches to sensitivity analyses would be using a different linear transformation in the cleavage prior, and using $\pi\left(\boldsymbol{\eta}_{F}\right)$ or $\pi\left(\boldsymbol{\eta}_{R}\right)$ rather than $\pi(\boldsymbol{\eta})$ in the sequence prior, and seeing whether the results changed much.

Our method is a promising addition to the peptide identification methodological toolbox.

\section{References}

Andrieu, C., de Freitas, N., Doucet, A., and Jordan, M. (2003). An introduction to MCMC for machine learning. Machine Learning, 50(1):5-43.

Ansong, C., Tolić, N., Purvine, S., Porwollik, S., Jones, M., Yoon, H., Payne, S., Martin, J., Burnet, M., Monroe, M., Venepally, P., Smith, R., Peterson, S., Heffron, F., McClelland, M., and Adkins, J. (2011). Experimental annotation of post-translational features and translated coding regions in the pathogen salmonella typhimurium. BMC Genomics, 12(1):433.

Bissiri, P. G., Holmes, C. C., and Walker, S. G. (2016). A general framework for updating belief distributions. Journal of the Royal Statistical Society Series B, 78(5):1103-1130.

Cleveland, J. P. and Rose, J. R. (2012). A neural network approach to the identification of b-/y-ions in MS/MS spectra. 012 IEEE International Conference on Bioinformatics and Biomedicine, 0:1-5.

Coombes, K. R., Baggerly, K. A., and Morris, J. S. (2007). Pre-processing mass spectrometry data. In Dubitzky, M. Granzow, M. and Berrar, D., editors, Fundamentals of Data Mining in Genomics and Proteomics, pages 79-99. Boston: Kluwer.

Damsleth, E. and El-Shaarawi, A. (1989). ARMA models with double-exponentially distributed noise. Journal of the Royal Statistical Society Series B, 51(1):61-69.

Dančík, V., Addona, T. A., Clauser, K. R., and Vath, J. E. (1999). De novo peptide sequencing via tandem mass spectrometry: A graph-theoretical approach. In RECOMB '99: Proceedings of the third annual international conference on Computational molecular biology, number 135-144, New York, NY, USA. ACM Press.

Diamandis, E. P. (2004). Mass spectrometry as a diagnostic and a cancer biomarker discovery tool: Opportunities and potential limitations. Molecular \& Cellular Proteomics, 3:367-378. 
Du, P., Stolovitzky, G., Horvatovich, P., Bischoff, R., Lim, J., and Suits, F. (2008). A noise model for mass spectrometry based proteomics. Bioinformatics, 24(8):1070-1077.

Frank, A. (2009). A ranking-based scoring function for peptide-spectrum matches. Journal of Proteome Research, 8(5):2241-2252.

Frank, A. and Pevzner, P. (2005). PepNovo: de novo peptide sequencing via probabilistic network modeling. Analytical Chemistry, 77(4):964-973.

Huang, Y., Triscari, J. M., Pasa-Tolic, L., Anderson, A. G., Lipton, M. S., Smith, R. D., and Wysocki, V. H. (2004). Dissociation behavior of doubly-charged tryptic peptides: Correlation of gas-phase cleavage abundance with ramachandran plots. Journal of American Chemical Society, 126:3034-3035.

IUBMB (1992). International Union of Biochemistry and Molecular Biology. In Liébecq, C., editor, Biochemical nomenclature and related documents. Portland Press, London. Second Edition.

Kemp, F. (2003). The Laplace distribution and generalizations: a revisit with applications to communications, economics, engineering, and finance. Journal of the Royal Statistical Society. Series D, 52(4):698-699.

Kirkpatrick, S., Gelatt, C. D., and Vecchi, M. P. (1983). Optimization by simulated annealing. Science, 220(4598):671680.

Lewis, C. N. (2013). Protein Identification Using Bayesian Stochastic Search. PhD thesis, University of South Carolina, Retrieved from http://scholarcommons.sc.edu/etd/2674.

Lubec, G. and Afjehi-Sadat, L. (2007). Limitations and pitfalls in protein identification by mass spectrometry. Chemical Reviews, 107(8):3568-3584.

Ma, B., Zhang, K., Hendrie, C., Liang, C., Li, M., Doherty-Kirby, A., and Lajoie, G. (2003). PEAKS: Powerful software for peptide de novo sequencing by tandem mass spectrometry. Rapid Communications in Mass Spectrometry, 17:2337-2341.

Robert, C. P. and Casella, G. (1999). Monte Carlo Statistical Methods. Springer-Verlag.

Rose, J. R., Cleveland, J. P., and Fox, A. (2010). An information theoretic approach to rescoring peptides produced by de novo peptide sequencing. International Conference on Bioinformatics and Computational Biology (Paris, France), World Academy of Science, Engineering and Technology, pages 200-205.

Schulze, W. (2004). Environmental proteomics - what proteins from soil and surface water can tell us: a perspective. Biogeosciences Discussions, 1:195-218.

Singla, P. and Domingos, P. (2005). Discriminative training of Markov logic networks. Proceedings of the 20th National Conference on Artificial Intelligence (AAAI), pages 868-873.

Sorensen, D. and Gianola, D. (2002). Likelihood, Bayesian and MCMC methods in quantitative genetics. Springer, New York.

Tierney, L. (1994). Markov chains for exploring posterior distributions. The Annals of Statistics, 22(4):1701-1728.

Visintin, I., Feng, Z., Longton, G., Ward, D. C., Alvero, A. B., Lai, Y., Tenthorey, J., Leiser, A., Flores-Saaib, R., Yu, H., Azori, M., Rutherford, T., Schwartz, P. E., and Mor, G. (2008). Diagnostic markers for early detection of ovarian cancer. Clinical Cancer Research, 14:1065-1072.

Wulfkuhle, J. D., Liotta, L. A., and Petricoin, E. F. (2003). Early detection: Proteomic applications for the early detection of cancer. Nature Reviews Cancer, 3:267-275.

Xu, C. and Ma, B. (2006). Software for computational peptide identification from MS-MS data. Drug Discovery Today, 11(13-14):595-600.

Yates, J. R., Ruse, C. I., and Nakorchevsky, A. (2009). Proteomics by mass spectrometry: approaches, advances, and applications. Annual Review of Biomedical Engineering, 11:49-79. 
Table 1: The top estimated peptides from the MCMC algorithm along with their corresponding log posterior densities, $\log$ of the scoring function value, $\log$ cleavage prior, $\log$ sequence prior, $\log \kappa_{1}$ prior, and $\log \kappa_{2}$ prior for the peptide $T G M S N V S K$ when using a simulated spectrum for each of the three noise levels using a Laplace noise structure.

\begin{tabular}{|c|c|c|c|c|c|c|c|}
\hline $\begin{array}{l}\text { Noise } \\
\text { Level }\end{array}$ & Peptide & $\begin{array}{l}\text { Log Posterior } \\
\text { Densities }\end{array}$ & $\begin{array}{c}\text { Log } \\
\text { Scoring Function Value }\end{array}$ & $\begin{array}{l}\text { Log Cleavage } \\
\text { Prior }\end{array}$ & $\begin{array}{l}\text { Log Sequence } \\
\text { Prior } \\
\end{array}$ & $\begin{array}{c}\log \kappa_{1} \\
\text { Prior }\end{array}$ & $\begin{array}{c}\log \kappa_{2} \\
\text { Prior }\end{array}$ \\
\hline \multirow{5}{*}{ Minimal } & TGMSNVSK & -42.58 & -12.84 & -8.03 & -25.62 & 5.30 & -1.40 \\
\hline & TGMYHSK & -47.51 & -20.86 & -7.51 & -22.00 & 4.37 & -1.51 \\
\hline & FADTIEK & -49.95 & -25.52 & -6.41 & -20.21 & 3.35 & -1.15 \\
\hline & WIFSDR & -50.66 & -28.08 & -4.84 & -20.35 & 3.82 & -1.20 \\
\hline & FVNNSDK & -54.76 & -26.37 & -6.98 & -22.18 & 2.15 & -1.39 \\
\hline \multirow{5}{*}{ Moderate } & TGMSNVSK & -27.82 & 0.98 & -8.94 & -25.62 & 6.08 & -0.32 \\
\hline & AEPTDYK & -47.97 & -24.68 & -5.39 & -21.15 & 3.76 & -0.50 \\
\hline & DEMLTSK & -49.36 & -24.43 & -5.85 & -22.76 & 4.17 & -0.49 \\
\hline & ASAYQQR & -49.39 & -22.53 & -8.26 & -21.49 & 3.37 & -0.48 \\
\hline & APNLAIPK & -51.56 & -22.29 & -8.97 & -22.70 & 2.94 & -0.54 \\
\hline \multirow{5}{*}{ Substantial } & TGMPFDR & 27.89 & 47.60 & -4.20 & -21.79 & 5.94 & 0.34 \\
\hline & TGMSNVTGG & 26.22 & 63.06 & -11.25 & -31.85 & 6.00 & 0.25 \\
\hline & TTSSNVASG & 16.59 & 49.34 & -7.83 & -31.11 & 6.00 & 0.19 \\
\hline & TGMSNVSQ & 14.27 & 41.50 & -5.23 & -28.29 & 6.04 & 0.25 \\
\hline & TGMSNVSK & 7.23 & 37.53 & -10.95 & -25.62 & 6.06 & 0.21 \\
\hline
\end{tabular}

Table 2: The top estimated peptides from the MCMC algorithm along with their corresponding log posterior densities, $\log$ of the scoring function value, $\log$ cleavage prior, $\log$ sequence prior, $\log \kappa_{1}$ prior, and $\log \kappa_{2}$ prior for the peptide $T G M S N V S K$ when using a simulated spectrum for each of the three noise levels using a Poisson noise structure.

\begin{tabular}{|c|c|c|c|c|c|c|c|}
\hline $\begin{array}{l}\text { Noise } \\
\text { Level }\end{array}$ & Peptide & $\begin{array}{l}\text { Log Posterior } \\
\text { Densities }\end{array}$ & $\begin{array}{c}\text { Log } \\
\text { Scoring Function Value }\end{array}$ & $\begin{array}{l}\text { Log Cleavage } \\
\text { Prior }\end{array}$ & $\begin{array}{l}\text { Log Sequence } \\
\text { Prior }\end{array}$ & $\begin{array}{l}\log \kappa_{1} \\
\text { Prior }\end{array}$ & $\begin{array}{c}\log \kappa_{2} \\
\text { Prior }\end{array}$ \\
\hline \multirow{5}{*}{ Minimal } & TGMSNVSK & -2.03 & 25.19 & -6.57 & -25.62 & 5.78 & -0.81 \\
\hline & TGMSRGSK & -24.68 & 8.33 & -6.50 & -31.16 & 5.33 & -0.67 \\
\hline & SVFNKTQ & -31.30 & -1.63 & -5.75 & -28.69 & 5.22 & -0.45 \\
\hline & TGMSRGSQ & -36.43 & 0.58 & -8.07 & -33.83 & 5.47 & -0.58 \\
\hline & NSVAAHPQ & -54.21 & -22.24 & -7.99 & -29.14 & 5.38 & -0.22 \\
\hline \multirow{5}{*}{ Moderate } & SAMSNVSK & 12.60 & 40.61 & -7.14 & -25.66 & 5.41 & -0.62 \\
\hline & TGMSNVSK & 11.30 & 43.79 & -11.66 & -25.62 & 5.33 & -0.55 \\
\hline & GTMSNVSK & 9.79 & 40.27 & -8.84 & -26.39 & 5.35 & -0.60 \\
\hline & SAFANVSK & 9.30 & 37.84 & -8.20 & -24.92 & 5.13 & -0.54 \\
\hline & TGMSNVSGA & -61.57 & -21.87 & -9.56 & -31.08 & 2.11 & -1.16 \\
\hline \multirow{5}{*}{ Substantial } & SAMSGGVSK & 17.97 & 49.44 & -7.79 & -28.35 & 5.01 & -0.34 \\
\hline & TGMSGGVSK & 14.31 & 46.91 & -8.81 & -28.31 & 4.85 & -0.34 \\
\hline & TGMSNVSK & 0.55 & 29.41 & -7.56 & -25.62 & 4.86 & -0.55 \\
\hline & TGMSNVSAG & -3.03 & 33.35 & -8.72 & -31.85 & 4.76 & -0.57 \\
\hline & TMGSNVSK & -7.21 & 21.85 & -7.56 & -25.62 & 4.82 & -0.70 \\
\hline
\end{tabular}


Table 3: The top estimated peptides from the MCMC algorithm along with their corresponding log posterior densities, $\log$ of the scoring function value, $\log$ cleavage prior, $\log$ sequence prior, $\log \kappa_{1}$ prior, and $\log \kappa_{2}$ prior for the peptide $Y H F E Q S T V T S Q P A R$ when using a simulated spectrum for each of the three noise levels using a Laplace noise structure.

\begin{tabular}{|c|c|c|c|c|c|c|c|}
\hline $\begin{array}{l}\text { Noise } \\
\text { Level }\end{array}$ & Peptide & $\begin{array}{l}\text { Log Posterior } \\
\text { Densities }\end{array}$ & $\begin{array}{c}\text { Log } \\
\text { Scoring Function Value }\end{array}$ & $\begin{array}{l}\text { Log Cleavage } \\
\text { Prior }\end{array}$ & $\begin{array}{l}\text { Log Sequence } \\
\text { Prior }\end{array}$ & $\begin{array}{c}\log \kappa_{1} \\
\text { Prior }\end{array}$ & $\begin{array}{c}\log \kappa_{2} \\
\text { Prior }\end{array}$ \\
\hline \multirow{5}{*}{ Minimal } & YHFEQSTVTSQPAR & 6.21 & 55.98 & -10.29 & -47.30 & 5.99 & 0.22 \\
\hline & YHFSEGSSVVSQPAR & 4.40 & 62.07 & -16.64 & -47.08 & 5.86 & 0.19 \\
\hline & YHFSEKTVTSQPAR & 3.60 & 55.12 & -10.29 & -47.30 & 5.82 & 0.25 \\
\hline & YHFSEGATTVSQPAR & 2.45 & 60.22 & -16.64 & -47.08 & 5.79 & 0.16 \\
\hline & LIAFFNGGGATCHEVD & -41.45 & 29.23 & -16.33 & -59.10 & 4.72 & 0.04 \\
\hline \multirow{5}{*}{ Moderate } & YHFEQSTVTSQPAR & 88.52 & 141.08 & -13.57 & -44.63 & 5.44 & 0.19 \\
\hline & YHFEQSTVTNTPVQ & 86.87 & 145.87 & -17.28 & -47.56 & 5.67 & 0.16 \\
\hline & GDKFEQSTVTSQPAR & 85.00 & 147.05 & -18.65 & -49.15 & 5.62 & 0.14 \\
\hline & YHFEQSTVTNTPAR & 84.01 & 134.36 & -12.12 & -43.85 & 5.53 & 0.09 \\
\hline & YHFAWSTVTSQPAR & 41.81 & 102.71 & -18.84 & -46.80 & 4.63 & 0.11 \\
\hline \multirow{5}{*}{ Substantial } & YHFEQSTVTQSPLN & 180.51 & 241.92 & -18.71 & -49.14 & 5.75 & 0.68 \\
\hline & YHFEKSTVTQSPAR & 163.42 & 220.25 & -14.52 & -48.20 & 5.16 & 0.73 \\
\hline & YHFEKSTVTSKPAR & 157.52 & 218.14 & -16.35 & -50.41 & 5.45 & 0.69 \\
\hline & YHFEQSTVTQSPAR & 154.39 & 206.48 & -13.09 & -44.69 & 5.02 & 0.67 \\
\hline & YHFEQSTSISQPAR & 116.96 & 169.27 & -12.00 & -45.25 & 4.39 & 0.55 \\
\hline
\end{tabular}

Table 4: The top estimated peptides from the MCMC algorithm along with their corresponding log posterior densities, $\log$ of the scoring function value, $\log$ cleavage prior, $\log$ sequence prior, $\log \kappa_{1}$ prior, and $\log \kappa_{2}$ prior for the peptide $Y H F E Q S T V T S Q P A R$ when using a simulated spectrum for each of the three noise levels using a Poisson noise structure.

\begin{tabular}{|c|c|c|c|c|c|c|c|}
\hline $\begin{array}{l}\text { Noise } \\
\text { Level }\end{array}$ & Peptide & $\begin{array}{c}\text { Log Posterior } \\
\text { Densities }\end{array}$ & $\begin{array}{c}\text { Log } \\
\text { Scoring Function Value }\end{array}$ & $\begin{array}{c}\text { Log Cleavage } \\
\text { Prior } \\
\end{array}$ & $\begin{array}{c}\text { Log Sequence } \\
\text { Prior } \\
\end{array}$ & $\begin{array}{c}\text { Log } \kappa_{1} \\
\text { Prior }\end{array}$ & $\begin{array}{c}\log \kappa_{2} \\
\text { Prior }\end{array}$ \\
\hline \multirow{5}{*}{ Minimal } & YHFEQSTVTSQPAR & 48.16 & 101.54 & -14.20 & -44.63 & 4.96 & 0.49 \\
\hline & YHFEQSTVTSKPAR & 44.39 & 100.61 & -14.65 & -46.89 & 4.88 & 0.44 \\
\hline & YHFEQSTVTSKPNL & 41.20 & 103.98 & -16.84 & -50.91 & 4.55 & 0.41 \\
\hline & YHFEQSTPCSPAGAR & -9.05 & 58.66 & -14.34 & -58.55 & 4.78 & 0.39 \\
\hline & YHFEKSPTSSSGPAR & -68.16 & 0.13 & -15.63 & -57.43 & 4.33 & 0.45 \\
\hline \multirow{5}{*}{ Moderate } & YHFQTFAVEGQPAVG & 8.55 & 74.36 & -17.51 & -52.15 & 3.76 & 0.10 \\
\hline & YHFEQSTVTSQPAR & 6.41 & 77.81 & -20.77 & -54.41 & 3.67 & 0.10 \\
\hline & YHFGATMEGEGQPAVG & -3.28 & 67.19 & -20.01 & -53.99 & 3.40 & 0.11 \\
\hline & YHFGAGMETEGQPAVG & -9.41 & 59.44 & -18.46 & -53.92 & 3.48 & 0.05 \\
\hline & YHFGATFAVEGQPAVG & -47.16 & 18.95 & -20.45 & -48.14 & 2.67 & -0.17 \\
\hline \multirow{5}{*}{ Substantial } & YHFEQSTVTSGAPAR & 140.22 & 192.03 & -17.16 & -47.31 & 5.92 & 0.40 \\
\hline & YHFEAGSTVTSGAPAR & 135.46 & 190.34 & -17.10 & -49.72 & 5.58 & 0.41 \\
\hline & YHFEAGSTVTSKPAR & 133.49 & 183.83 & -13.50 & -49.31 & 5.87 & 0.36 \\
\hline & YHFEQSTVTSQPAR & 128.28 & 173.90 & -13.44 & -44.63 & 5.86 & 0.37 \\
\hline & YHFEQSTVMGQPAR & 116.28 & 165.56 & -15.99 & -45.28 & 5.66 & 0.33 \\
\hline
\end{tabular}

Table 5: The top estimated peptides from the MCMC algorithm along with their corresponding log posterior densities, $\log$ of the scoring function value, $\log$ cleavage prior, $\log$ sequence prior, $\log \kappa_{1}$ prior, and $\log \kappa_{2}$ prior for the peptide $T G M S N V S K$. The true peptide is in bold.

\begin{tabular}{|c|c|c|c|c|c|c|}
\hline Peptide & $\begin{array}{c}\text { Log Posterior } \\
\text { Densities }\end{array}$ & $\begin{array}{c}\text { Log } \\
\text { Scoring Function Value }\end{array}$ & $\begin{array}{c}\text { Log Cleavage } \\
\text { Prior }\end{array}$ & $\begin{array}{c}\text { Log Sequence } \\
\text { Prior }\end{array}$ & $\begin{array}{c}\text { Log } \kappa_{1} \\
\text { Prior }\end{array}$ & $\begin{array}{c}\text { Log } \kappa_{2} \\
\text { Prior }\end{array}$ \\
\hline TGMSNVSK & -15.16 & 12.87 & -8.17 & -25.62 & 6.36 & -0.60 \\
\hline TMGSNVSK & -44.00 & -16.19 & -7.18 & -25.75 & 5.91 & -0.79 \\
\hline TWSNSTK & -47.16 & -21.11 & -6.02 & -23.96 & 4.47 & -0.54 \\
\hline FDIGDTR & -49.74 & -24.43 & -6.94 & -21.34 & 3.61 & -0.64 \\
\hline WEMDDK & -49.76 & -26.55 & -6.42 & -19.54 & 3.36 & -0.61 \\
\hline VYTLSLK & -52.27 & -27.88 & -4.70 & -22.09 & 2.90 & -0.50 \\
\hline YLTGLEK & -56.91 & -32.42 & -6.32 & -21.32 & 3.59 & -0.43 \\
\hline FFPAVSR & -56.95 & -30.52 & -7.87 & -20.88 & 3.06 & -0.74 \\
\hline TMEEVSK & -57.88 & -35.85 & -4.69 & -22.42 & 5.67 & -0.60 \\
\hline VSTYLLK & -58.16 & -31.97 & -7.13 & -21.46 & 3.11 & -0.71 \\
\hline
\end{tabular}


Table 6: The top estimated peptides from the MCMC algorithm along with their corresponding log posterior densities for the peptide TGMSNVSK for three different starting peptides. The true peptide is in bold.

\begin{tabular}{|c|c|c|c|c|c|}
\hline \multicolumn{6}{|c|}{ Starting Peptide } \\
\hline \multicolumn{2}{|c|}{ SAMYHSK } & \multicolumn{2}{|c|}{ TGAFGRSK } & \multicolumn{2}{|c|}{ GTFANEGK } \\
\hline Peptide & Log Posterior & Peptide & Log Posterior & Peptide & Log Posterior \\
\hline TGMSNVSK & -14.31 & TGMSNVSK & -17.95 & TGMSNVSK & -14.67 \\
\hline SMTQTQK & -49.50 & ITLYADK & -27.54 & MGSNVSQ & -22.76 \\
\hline TMTLETQ & -50.12 & TGMVTTTL & -31.73 & TFQSQWK & -36.20 \\
\hline SLSIYLK & -52.39 & MGAITMSI & -41.43 & VMDPFSK & -40.15 \\
\hline GYDNLNK & -54.01 & CGMEAANK & -44.11 & AYIISEK & -41.73 \\
\hline EATFDLK & -55.05 & FLVDAMK & -44.76 & YGPETEK & -47.05 \\
\hline HFPIPGR & -55.601 & TGMSDVLT & -51.78 & TWYVVR & -48.64 \\
\hline YDQPATE & -56.13 & FFGLIAR & -51.88 & DVFDSLK & -48.89 \\
\hline GAQYGEAK & -57.43 & TGMSLTTL & -52.84 & WHLPDR & -49.39 \\
\hline WTYLLQ & -58.16 & -ITLYVSK & -52.10 & TGAFNWK & -57.68 \\
\hline
\end{tabular}

Table 7: The top estimated peptides from the MCMC algorithm along with their corresponding log posterior densities, $\log$ of the scoring function value, $\log$ cleavage prior, $\log$ sequence prior, $\log \kappa_{1}$ prior, and $\log \kappa_{2}$ prior for the peptide $D L V E S A P A A L K$

\begin{tabular}{|c|c|c|c|c|c|c|}
\hline Peptide & $\begin{array}{c}\text { Log Posterior } \\
\text { Densities }\end{array}$ & $\begin{array}{c}\text { Log } \\
\text { Scoring Function Value }\end{array}$ & $\begin{array}{c}\text { Log Cleavage } \\
\text { Prior }\end{array}$ & $\begin{array}{c}\text { Log Sequence } \\
\text { Prior }\end{array}$ & $\begin{array}{c}\text { Log } \kappa_{1} \\
\text { Prior }\end{array}$ & $\begin{array}{c}\text { Log } \kappa_{2} \\
\text { Prior }\end{array}$ \\
\hline DLVESAPAALK & 42.90 & 78.94 & -11.25 & -31.26 & 6.31 & 0.15 \\
\hline AVQIEQVQAQ & 8.56 & 47.48 & -8.57 & -33.83 & 3.51 & -0.03 \\
\hline DLVESPAAAIK & 2.63 & 40.03 & -11.28 & -31.69 & 5.435 & 0.14 \\
\hline LTELASPAALQ & -0.94 & 38.74 & -10.70 & -34.13 & 4.92 & 0.23 \\
\hline DLVESAPAAIK & -1.65 & 35.86 & -11.28 & -31.69 & 5.38 & 0.07 \\
\hline QVDIVLGDVR & -7.24 & 30.33 & -11.50 & -29.70 & 3.42 & 0.21 \\
\hline QVDIIVDGVR & -14.54 & 23.36 & -12.99 & -29.67 & 4.61 & 0.16 \\
\hline EVPATTGGVGPE & -16.30 & 29.28 & -12.53 & -37.61 & 4.52 & 0.04 \\
\hline QVDIPDDGVR & -16.89 & 21.68 & -12.99 & -29.67 & 4.15 & -0.04 \\
\hline EVPTATGGVGPE & -17.22 & 27.76 & -12.53 & -37.61 & 4.89 & 0.27 \\
\hline
\end{tabular}

Table 8: The top estimated peptides from the MCMC algorithm along with their corresponding log posterior densities for the peptide $D L V E S A P A A L K$ for three different starting peptides. The true peptide is in bold.

\begin{tabular}{|c|c||c|c||c|c|}
\hline \multicolumn{2}{|c||}{ Starting Peptide } \\
\hline Deptide & Log Posterior & Peptide & Log Posterior & \multicolumn{2}{c|}{ LDVETGPAALK } \\
\hline DDLTVAAPAIK & 30.35 & DLVESAPAALK & 31.51 & DLVDTAde & Log Posterior \\
\hline DLVESAPAALK & 21.05 & VADPANGQLTQ & 11.97 & DLVESAPAALK & 35.76 \\
\hline AVSPAWGNLAK & 14.49 & SPFSISYANK & -5.26 & LDVDVAGAPEK & 7.25 \\
\hline MVIVTAAPLAK & 3.01 & DQSNDYDEK & -8.41 & LDVTIAGAPEK & -5.06 \\
\hline DLTPVSVSPAK & -1.97 & DLVESAPIAAK & -8.81 & APVDIIQDDK & -7.49 \\
\hline DDLLGTAPALK & -4.03 & DAEDMYLK & -9.49 & DLVDTAPAALK & -9.00 \\
\hline NNVEASPAALK & -5.12 & ELEGNEPMPVK & -9.64 & MDMMEDLTK & -15.49 \\
\hline DPVDWVAALK & -6.57 & VFPLSPDNPK & -16.21 & DLLTTAPAALK & -19.25 \\
\hline DDLVTAAPAIK & -15.07 & DVLASEPASPK & -23.99 & DLLATTPAALK & -20.61 \\
\hline DIVDENPLAK & -15.66 & DISIDPALGGR & -24.47 & EYTIEFIAK & -21.00 \\
\hline
\end{tabular}


Table 9: The best estimated peptides using the PepNovo Rank Score, PepNovo Score, and our method (Bayesian posterior). The last column is the true peptide. The number of switches it takes to obtain the true peptide is in parentheses. (0) denotes the estimated peptide is the true peptide. (*) denotes that the minimum number of switches cannot be found since the estimated peptide does not have the correct total mass.

\begin{tabular}{|c|c|c||c|}
\hline \multicolumn{2}{|c|}{ Best Estimated Peptides } & True Peptide \\
\hline PepNovo Rank Score & PepNovo Score & Bayes Log Posterior & DLVESAPAALK \\
\hline DNVESLEV $(*)$ & DLVESAPAALK $(0)$ & DLVESAPAALK $(0)$ & AQLQEIAQTK \\
\hline AQLQNQAQTK $(1)$ & VVLQELAQTK $(1)$ & AQLQEIAQTK $(0)$ & SVANAEQMDR \\
\hline SVANAEQMDR $(0)$ & WANAQEMDR $(2)$ & SVANAEQMDR $(0)$ & SILSELVR \\
\hline AELSELV $(*)$ & VTLSELVR $(1)$ & SIISELVR $(0)$ & TGMSNVSK \\
\hline TGMSNVSK $(0)$ & TGMSNVSK $(0)$ & TGMSNVSK $(0)$ & VSEGQTVR \\
\hline VSEGQTVR $(0)$ & VSEGQTVR $(0)$ & VSEGQTVR $(0)$ & SGPLAGYPVVDLGVR \\
\hline QASEVVSLNK $(*)$ & FEHAAASEVVSLGGK $(3+)$ & SGPLAGYPVVDIGVR $(0)$ & YHFEQSTVTSQPAR \\
\hline SQESTVTSQPAR $(*)$ & YHFEQESATSQVPK $(2)$ & YHFEQSTVTSQAPR $(1)$ & MPPTEGETGGQVLGSK \\
\hline MPPTEGETNQVL $(*)$ & MPPTEGETNQVLGSK $(1)$ & MPPTEGETGGQVLGSK $(0)$ & GYAGDTATTSEVK \\
\hline GYAGDTATTSEVK $(0)$ & GYAGDTATTSEVK $(0)$ & GYAGDTATTSEVK $(0)$ & LVSSPSTLNPGTNVAK \\
\hline NPSSPSDVLSS $(*)$ & VLSSPSDPDSPVQEK $(3+)$ & LVSSPSTLNPGTNVAK $(0)$ & AFNEALPLTGVVLTK \\
\hline LPDVGVVLTK $(*)$ & NTVFALVLVLAALTK $(3+)$ & QWFSPPLVTGVITK $(3+)$ &
\end{tabular}

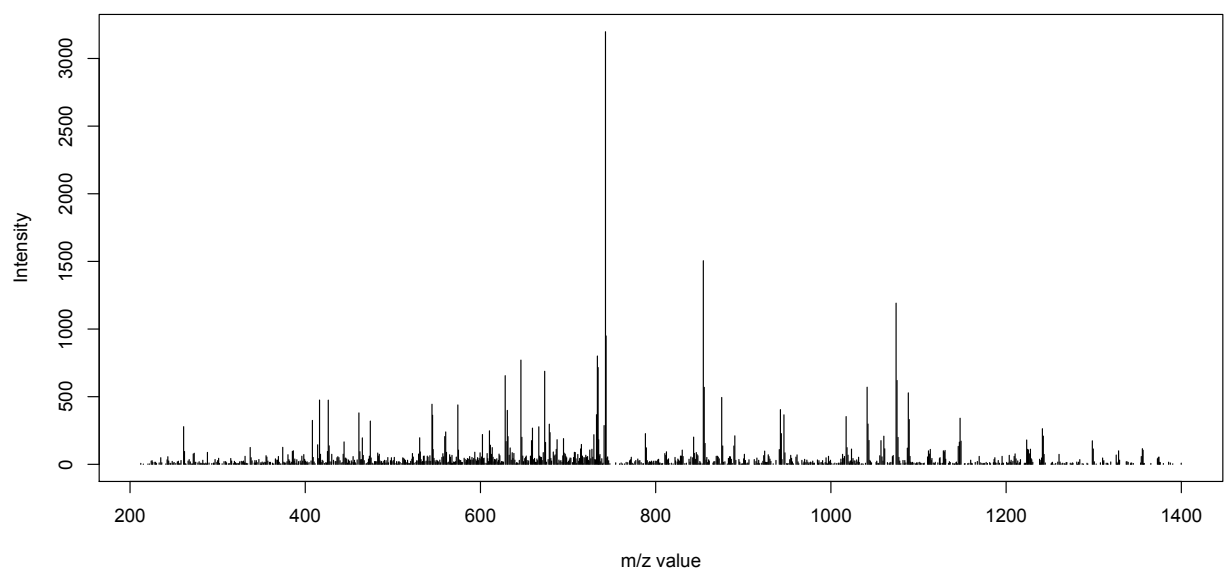

Figure 1: Line plot of pairs of intensities and $m / z$ values for a given peptide.

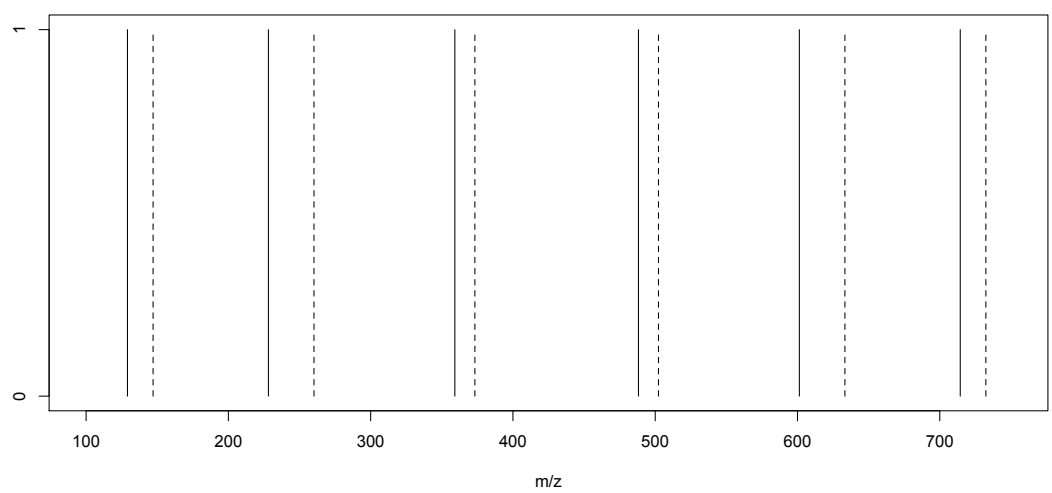

Figure 2: Theoretical spectrum for the peptide $Q V M E L L Q$ using only $b$ and $y$ ions. Here 1 represents the presence of an ion and 0 represents the absence of an ion. The $b$ ion is denoted by solid lines and the $y$ ion is denoted by dashed lines. 


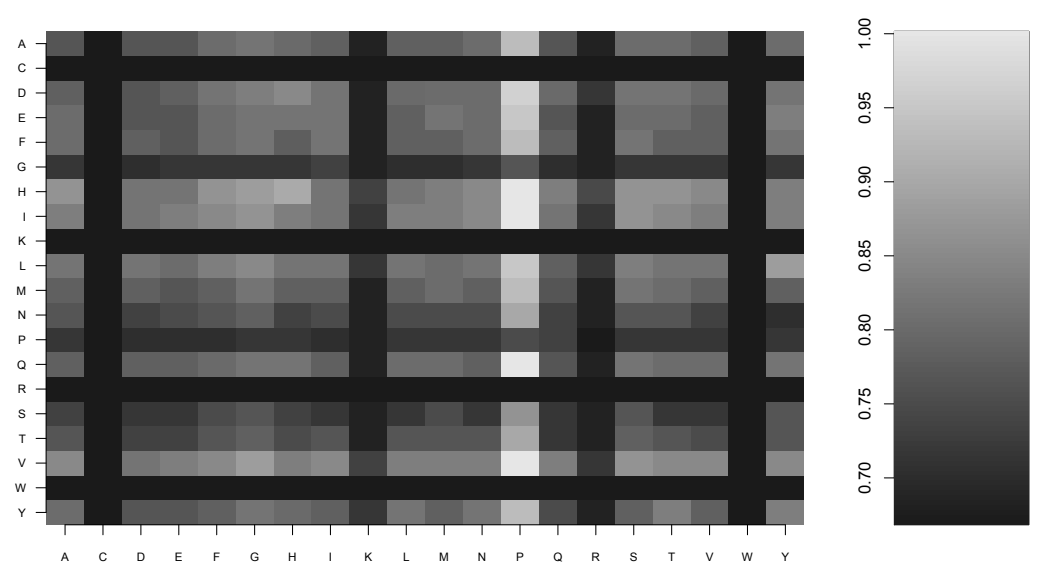

Figure 3: A display of the geometric mean of average relative abundances of bond cleavages of $b$ and $y$ ions for any particular amino acid pair using Figure 1 in Huang et al. (2004). The y-axis is the single letter code of the amino acid on the $\mathrm{N}$-terminal amino acid and the $\mathrm{x}$-axis is the single letter code of the amino acid on the C-terminal amino acid.
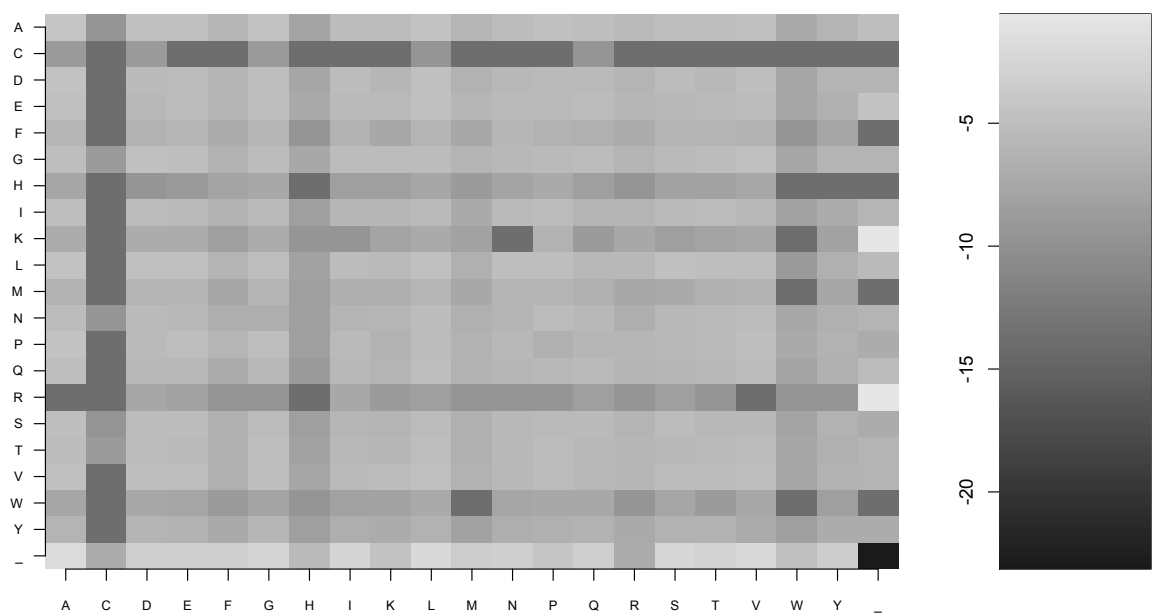

Figure 4: The joint empirical probabilities for all pairs of amino acids. For ease of identifying the different joint empirical probabilities, the figure is shown on the log-scale. The y-axis is the single letter code of the amino acid for the first amino acid in the pair and the $\mathrm{x}$-axis is the single letter code of the amino acid for the second amino acid in the pair. The darker the square, the less probable the pair. 


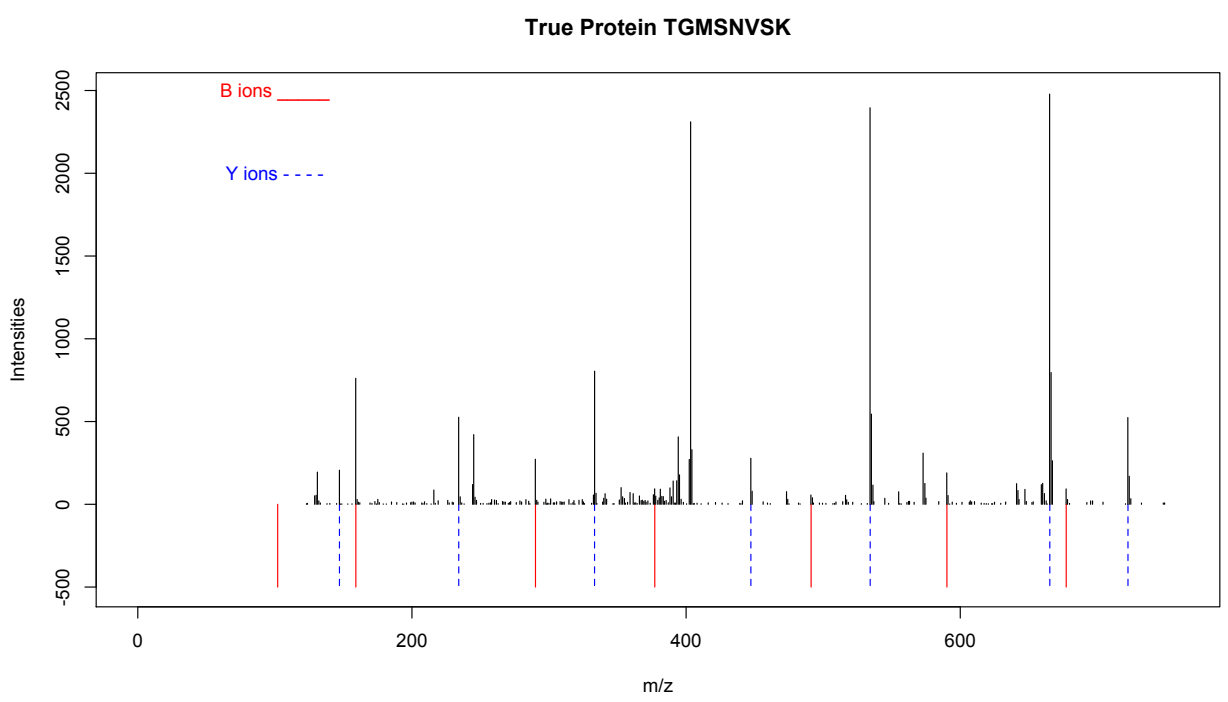

Figure 5: The observed spectrum plotted against the theoretical spectrum for the peptide TGMSNVSK. The theoretical spectrum is plotted below the zero axis and the observed spectrum is plotted above the zero axis.

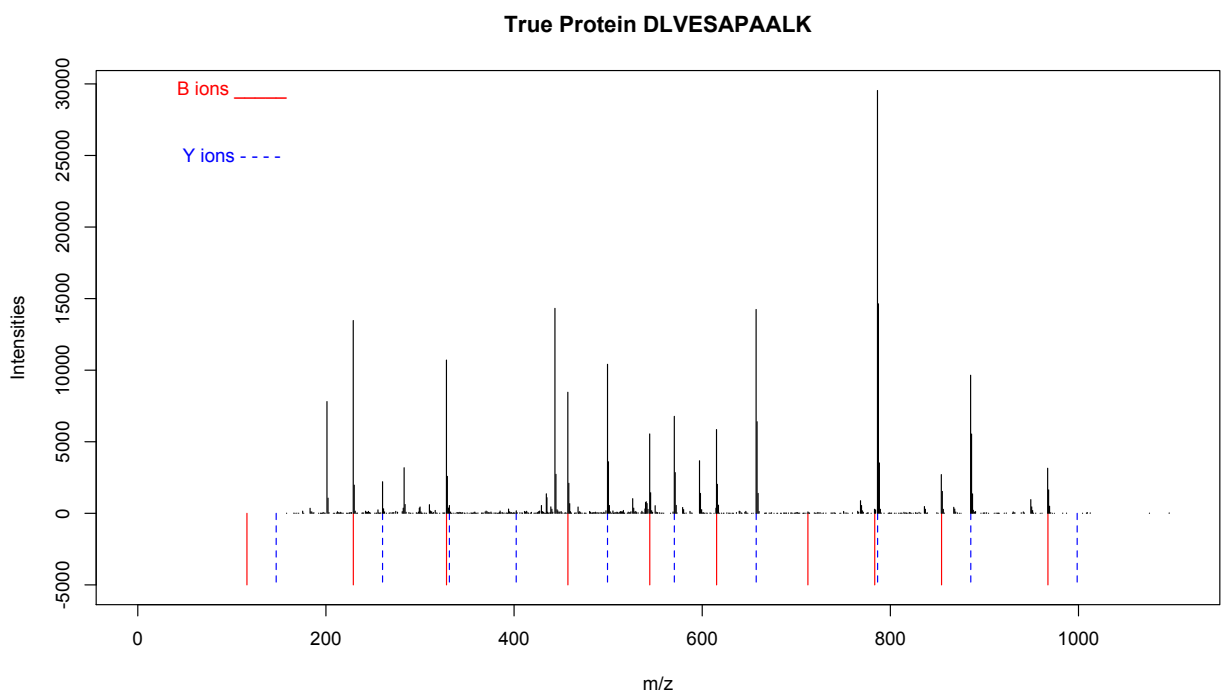

Figure 6: The observed spectrum plotted against the theoretical spectrum for the peptide DLVESAPAALK. The theoretical spectrum is plotted below the zero axis and the observed spectrum is plotted above the zero axis. 